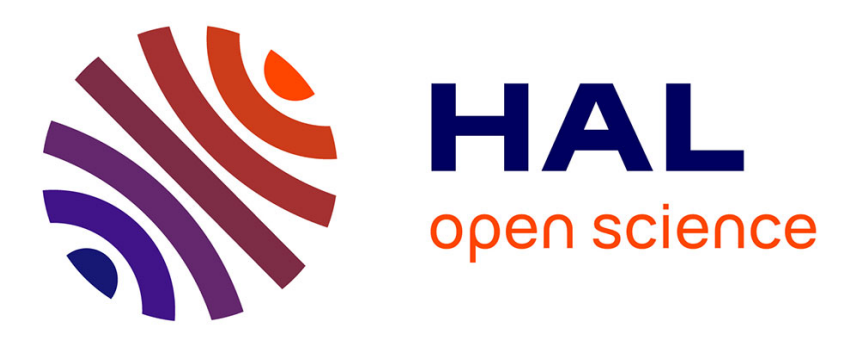

\title{
Sticking Probability of Ammonia Molecules on Tungsten and 316L Stainless Steel Surfaces
}

\author{
M. Minissale, J.-B. Faure, A. Dunand, T. Angot, G. de Temmerman, R.
} Bisson

\section{> To cite this version:}

M. Minissale, J.-B. Faure, A. Dunand, T. Angot, G. de Temmerman, et al.. Sticking Probability of Ammonia Molecules on Tungsten and 316L Stainless Steel Surfaces. Journal of Physical Chemistry C, 2020, 124 (32), pp.17566-17577. 10.1021/acs.jpcc.0c03313 . hal-03046773

\section{HAL Id: hal-03046773 \\ https://hal-amu.archives-ouvertes.fr/hal-03046773}

Submitted on 20 Apr 2021

HAL is a multi-disciplinary open access archive for the deposit and dissemination of scientific research documents, whether they are published or not. The documents may come from teaching and research institutions in France or abroad, or from public or private research centers.
L'archive ouverte pluridisciplinaire HAL, est destinée au dépôt et à la diffusion de documents scientifiques de niveau recherche, publiés ou non, émanant des établissements d'enseignement et de recherche français ou étrangers, des laboratoires publics ou privés. 


\title{
The Sticking Probability of Ammonia Molecules
}

\section{on Tungsten and 316L Stainless Steel Surfaces}

\author{
M. Minissale,${ }^{\dagger}$ J.-B. Faure,${ }^{\dagger}$ A. Dunand,${ }^{\dagger}$ T. Angot, ${ }^{\dagger}$ G. De Temmerman,${ }^{\dagger}$ and \\ R. Bisson*, ${ }^{*}$
}

$\dagger$ Aix-Marseille Univ, CNRS, PIIM, Marseille, France

$\$$ †TER Organization, Route de Vinon-sur-Verdon, CS 90 046, 13067 St Paul Lez Durance Cedex,

France

E-mail: regis.bisson@univ-amu.fr 


\begin{abstract}
We present measurements of the sticking probability of ammonia on two metals, tungsten and 316L stainless steel, covered with natural surface impurities as they will be used for the international nuclear fusion experimental reactor ITER. By using a collimated supersonic molecular beam at two different kinetic energies (55 and $255 \mathrm{meV}$ ), varying the sample temperature in the $130-425 \mathrm{~K}$ range and characterizing the surface composition with Auger electron spectroscopy, we observe similar sticking features on both surfaces consistent with a non-dissociative adsorption mediated by two precursors having different trapping probabilities. First, the initial sticking probability decreases with increasing the surface temperature. Second, the sticking probability increases with the surface coverage up to near-saturation coverage where it declines. Both features cannot be described together with the Kisliuk model (intrinsic + extrinsic precursors with identical trapping probabilities) nor the modified Kisliuk model (direct adsorption + extrinsic precursor). Thus, we derive a Generalized and Separable Kisliuk (GSK) model that is able to reproduce quantitatively these two experimental observations thanks to intrinsic and extrinsic precursors having different trapping probabilities. The GSK model assumes a negligible transfer from the intrinsic precursor to the extrinsic precursor which allows to extract precursors kinetics parameters in a two step analysis. The GSK analysis indicates that ammonia trapping probability is lower on the bare surface (intrinsic precursor) than on the $\mathrm{NH}_{3}$ covered surface (extrinsic precursor). Furthermore, the barriers between the two precursors wells and the deep adsorption well are found below the vacuum level. Finally, we measure that the sticking probability does not decline to zero i.e. steady-state sticking is observed with a probability up to 0.15 at a beam energy of $55 \mathrm{meV}$ and a surface temperature of $220 \mathrm{~K}$. This observation is consistent with $\mathrm{NH}_{3}$ multi-layer adsorption. These experimental results and their fitting with the GSK model offer the starting point to a predictive determination of the fusion fuel inventory related to ammonia in the international experimental reactor ITER.
\end{abstract}




\section{Introduction}

Efficient ammonia $\left(\mathrm{NH}_{3}\right)$ production is a story of scientific ${ }^{1}$, technological and human progresses ${ }^{2}$. It is estimated that about a third of the population growth in the twentieth century was possible thanks to the Haber-Bosch process, which produces $\mathrm{NH}_{3}$ molecules on the surface of iron-based catalysts at high pressure $\left(\sim 10^{7} \mathrm{~Pa}\right)$ and relatively high temperature $(\sim 700 \mathrm{~K}) . \mathrm{NH}_{3}$ production being responsible for $\sim 2 \%$ of the world's consumption of fossil fuels, there is currently worldwide efforts to find alternatives to the Haber-Bosch process ${ }^{3}$. Nevertheless, $\mathrm{NH}_{3}$ production is of current concern in another technological endeavor, namely, the international nuclear fusion experimental reactor ITER under construction in France. In ITER, a fuel mixture of hydrogen isotopes, deuterium and radioactive tritium, will be heated and magnetically confined in a vacuum vessel to form a hot plasma producing $500 \mathrm{MW}$ of fusion power. Power exhaust is mainly performed in the so-called divertor region, a special area of the plasma chamber where the open magnetic field lines intersect the primary plasma facing components and where the plasma is neutralized and pumped away. Tungsten (W) is used as plasma-facing material while the support structure is made of a variant (316L(N)-IG) of 316L stainless steel (316L-SS). In order to control the steady-state power density within tolerable limits (typically on the order of $\sim 10 \mathrm{MW} \cdot \mathrm{m}^{-2}$ ), it is necessary to dissipate a large part of the exhaust power by collision-induced isotropic radiation of intentionally injected impurities. To date, the best compromise between radiative efficiency ${ }^{4}$ and hot plasma performance ${ }^{5-7}$ has been achieved with the injection of molecular nitrogen in the divertor plasma region. However, injection of nitrogen in a divertor plasma environment next to metallic components leads to the production of $\mathrm{NH}_{3}$ with a conversion efficiency for the injected $\mathrm{N}_{2}$ on the order of $10 \%{ }^{8-10}$. In ITER, ammonia production will be concurrent with a deuterium/tritium plasma thus radioactive tritiated ammonia is expected. It is well known that ammonia is a polar molecule and it could stick on shadowed metallic in-vessel components (W and 316L-SS) as well as on exhaust pumping ducts (316L-SS). This represents a risk of accumulation of tritiated species in remote areas in addition to a duty-cycle issue for the tritium recycling plant operated to retrieve tritium from cryo-pumps and exhaust gases. For all these reasons, it is mandatory to estimate the sticking probability $(S)$ of 
$\mathrm{NH}_{3}$ on $\mathrm{W}$ and $316 \mathrm{~L}-\mathrm{SS}$.

The interaction of $\mathrm{NH}_{3}$ with $\mathrm{W}$ has been studied since the 1920 s but a detailed understanding of its behavior on clean surfaces appeared in the 1980s with the advent of surface sensitive spectroscopies ${ }^{11,12}$ and molecular beam techniques ${ }^{13-15}$. Grunze et al. on clean $\mathrm{W}(110)^{11}$ and Egawa et al. on clean W(100) ${ }^{12}$ measured both with X-ray photoelectron spectroscopy (XPS) and ultraviolet photoelectron spectroscopy (UPS) that $\mathrm{NH}_{3}$ adsorbs molecularly below $120 \mathrm{~K}$. $\mathrm{NH}_{3}$ dissociates in $\mathrm{NH}_{2}$ and $\mathrm{H}$ when the temperature is increased, at $170 \mathrm{~K}$ on $\mathrm{W}(110)$ or at $270 \mathrm{~K}$ on W(100). Further dissociation in NH fragment is observed at $300 \mathrm{~K}$ on W(110) and W(100). Additionally, $\mathrm{NH}_{3}$ desorption between $200 \mathrm{~K}$ and $400 \mathrm{~K}$ was observed by mass spectrometry on both surfaces ${ }^{13-15}$. Thus, desorption and dissociation are competitive processes on clean $\mathrm{W}$. The origin of this competition was understood thanks to the measurements of the sticking probability of $\mathrm{NH}_{3}$ with molecular beam techniques. Alnot et al. used a collimated effusive (thermal) molecular beam and measured on $\mathrm{W}(100)$ an initial sticking probability $\left(S_{0}\right)$ of $\mathrm{NH}_{3}$ at normal incidence of 1.0 at $320 \mathrm{~K}$ that decreases to 0.6 at $910 \mathrm{~K}^{14}$. This result is consistent with a precursor model where impinging $\mathrm{NH}_{3}$ molecules are first trapped in a molecular adsorption well before to desorb or to break apart in a dissociative adsorption well. Masson et al. used a collimated supersonic molecular beam to measure $S_{0}$ as a function of $\mathrm{NH}_{3}$ kinetic energy $\left(E_{k}\right)$ and surface temperature $\left(T_{s}\right)$ on $\mathrm{W}(110)$. They found that, for $E_{k}=26 \mathrm{meV}, S_{0}$ decreases when increasing $T_{s}$, from 1.0 at $200 \mathrm{~K}$ to 0.1 at $650 \mathrm{~K}$. Furthermore, for $T_{s}=150 \mathrm{~K}, S_{0}$ decreases when increasing $E_{k}$, from 1.0 at $E_{k}=26 \mathrm{meV}$ to 0.55 at $E_{k}=234 \mathrm{meV}$. Again, this is consistent with a precursor model even though Masson et al. needed to use a precursor model with two molecular adsorption wells, one being possibly partially dissociative, preceding a totally dissociative well to fit their entire data set.

Of interest for the present work on W, we stress that Grunze et al. has also studied spectroscopically the effect of impurities onto $\mathrm{NH}_{3}$ adsorption ${ }^{11}$. First, they were able to detect the growth of a second layer of $\mathrm{NH}_{3}$ at $T_{s}=80 \mathrm{~K}$ when W(110) was saturated with ammonia. Second, they pre-covered W(110) with 0.2 monolayer of oxygen and found that it reduces the dissociative rate of $\mathrm{NH}_{3}$ at $T_{s}=300 \mathrm{~K}$ as well as stabilizes the molecular adsorption of intact $\mathrm{NH}_{3}$. 
Regarding the interaction of $\mathrm{NH}_{3}$ with $316 \mathrm{~L}-\mathrm{SS}$, no systematic work was found in the literature, even though the ammonia stickiness on this surface has been recognized to be perturbing from the experimental point of view for at least 40 years ${ }^{16,17}$. Recently, de Castro et al. estimated sticking probabilities of $\mathrm{NH}_{3}$ on 304-L stainless steel ${ }^{18}$ on the order of $0.08-0.04$ in the temperature range 323 - $473 \mathrm{~K}$. However, these measurements were realized in a vessel at relatively high pressure $\left(10^{-2}\right.$ mbar $)$ and therefore do not concern the low coverage limit. These authors also estimated that thousands of $\mathrm{NH}_{3}$ layers should build up in the $10^{-2}$ mbar range near room temperature. However, this estimation was contradicted by the work of Yaala et al. ${ }^{19}$ using quartz micro-balance measurements. In this recent study, it was measured that $\mathrm{NH}_{3}$ multi-layer build up at room temperature, both for polycrystalline tungsten and stainless steel, reaching saturation value between 2 and 20 monolayers at, respectively, $10^{-3}$ mbar and 800 mbar. Nevertheless, it was not attempted to extract sticking probabilities from this data.

In the present work, we performed a systematic study of the sticking probability of $\mathrm{NH}_{3}$ on polycrystalline $\mathrm{W}$ and 316L stainless steel in the state in which they will be used in the ITER reactor, i.e. on surfaces that are not atomically clean but contain impurities. This way we gained knowledge on the dynamics and the kinetics of $\mathrm{NH}_{3}$ adsorption and desorption on technologically relevant surfaces.

\section{Experimental Section}

\subsection{The AMU-PSI set-up}

Experiments were performed in the Advanced MUltibeam experiments for Plasma Surface Interaction (AMU-PSI) at Aix-Marseille University (Marseille, France). AMU-PSI is a cylindrical 2-stories ultra-high vacuum (UHV) set-up, projected on a single storey in Figure 1, dedicated to the investigation of fundamental plasma-surface interactions. It is composed of a UHV sample chamber (base pressure $2 \times 10^{-10}$ mbar) equipped with a 4-axis sample manipulator, a 4-grid low energy electron diffractometer / Auger electron spectrometer (OCI), a high-flux ion beam (Focus, 
not used in the present study), a home-made supersonic molecular beam and a quadrupole mass spectrometer (MKS Microvision2). An actively cooled oven is installed on the sample manipulator for in situ sample preparation and allows to cover the range 120 - $2300 \mathrm{~K}$. The sample temperature is measured via a C-type thermocouple mechanically pressed on the sample and it has been calibrated against boiling liquid nitrogen and boiling water for the low temperature range.

To gain insights into $\mathrm{NH}_{3}$ adsorption and desorption on metallic surfaces, we varied two experimental parameters: the sample surface temperature $\left(T_{s}\right)$ and the kinetic energy of $\mathrm{NH}_{3}$ molecules $\left(E_{k}\right)$. Depending on how sticking probabilities evolve with these two parameters, one can determine what is the type of dynamical mechanism that leads to adsorption and extract kinetics information about potential energy wells and activation energy barriers ${ }^{20}$. In the present study, the surface temperature was varied between $130 \mathrm{~K}$ and $425 \mathrm{~K}$. For temperature below $295 \mathrm{~K}$, a combination of liquid nitrogen cooling and radiative heating was used. For temperature at or above 295 $\mathrm{K}$, only radiative heating was used. For the variation of the kinetic energy, we seeded $\mathrm{NH}_{3}$ in a carrier gas as it will be detailed in the following section.

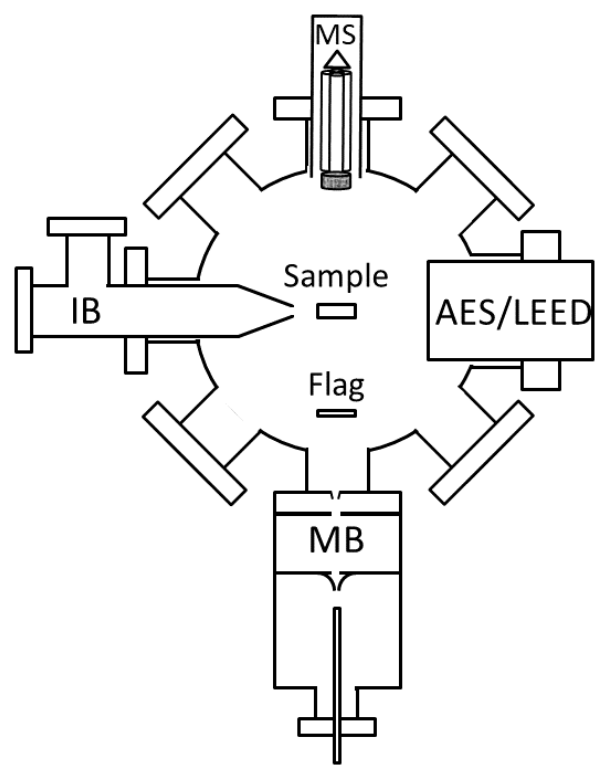

Figure 1: Schematic top-view of the AMU-PSI set-up. MB: Supersonic molecular beam. MS: Quadrupole mass spectrometer. AES/LEED: Auger electron spectrometer and low energy electron diffractometer. IB: Ion beam, not used in the present study. 


\subsection{Supersonic molecular beam}

The sample chamber is connected to a triply differentially pumped supersonic molecular beam that is collimated to the center of the sample chamber and delivers $\mathrm{NH}_{3}$ to the sample surface with well defined incidence angle and kinetic energy. The molecular beam spot diameter of $\sim 5 \mathrm{~mm}$ is defined by the geometric configuration illustrated in Figure 1, in particular by the $50 \mu \mathrm{m}$ hole of the gas source nozzle and the $2 \mathrm{~mm}$ conical aperture separating the third molecular beam stage from the sample chamber.

The mean kinetic energy $\left(E_{k}\right)$ of $\mathrm{NH}_{3}$ molecules in the molecular beam was set by seeding $2 \%$ of $\mathrm{NH}_{3}$ in a carrier gas, $\mathrm{He}$ or $\mathrm{N}_{2}$. The gas purity grade is $6.0(99.9999 \%)$ with a $5 \%$ relative incertitude on the $\mathrm{NH}_{3}$ content (Messer). The mean kinetic energy was calculated considering an ideal gas expansion of the binary mixture, which applies in the case of the free-jet supersonic expansion we used in the present studies. $E_{k}$ was calculated using the formula

$$
E_{k}=\frac{\bar{\gamma}}{\bar{\gamma}-1} \frac{m}{\bar{m}} k_{b} T_{n}
$$

where $\bar{\gamma}$ is the average ratio of constant pressure to constant volume specific heat of the mixture, $m$ is the mass of the ammonia molecule, $\bar{m}$ is the average mass of the mixture, $k_{b}$ is the Boltzmann constant and $T_{n}$ is the gas source nozzle temperature. A list of the parameters used here is given in table $1 . \mathrm{NH}_{3}$ in $\mathrm{He}$ was jet expanded at room temperature to obtain a mean kinetic energy of $\sim 255$ meV, while $\mathrm{NH}_{3}$ was seeded in $\mathrm{N}_{2}$ in the same condition to obtain a mean kinetic energy of $\sim 55$ $\mathrm{meV}$.

Table 1: Parameters used to calculate the kinetic energy of $\mathrm{NH}_{3}$.

\begin{tabular}{lccccc}
\hline \hline & $\gamma_{N_{3}}$ & $\gamma_{C G}^{a}$ & $\bar{\gamma}$ & $\bar{m}^{b}$ & $E_{k}^{c}(\mathrm{meV})$ \\
\hline $\mathrm{NH}_{3} / \mathrm{N}_{2}$ & 1.333 & 1.400 & 1.399 & 27.79 & 55 \\
$\mathrm{NH}_{3} / \mathrm{He}$ & 1.333 & 1.667 & 1.660 & 4.26 & 255 \\
\hline \hline${ }^{a} \mathrm{CG}=$ Carrier Gas \\
${ }^{b}$ Calculated for $2 \%$ of $\mathrm{NH}_{3}$ in the $\mathrm{CG}$. \\
${ }^{c}$ Calculated for $T_{n}=295 \mathrm{~K}$.
\end{tabular}


The $\mathrm{NH}_{3}$ molecular beam flux is defined by three parameters: the pressure of the binary gas mixture source, the pressure in the supersonic free-jet expansion chamber and the distance between the source and the skimmer (Beam Dynamics model 2) that extract the molecular beam from the jet expansion. In the present study, the source pressure was set to $1.2 \mathrm{bar}$, the pressure in the expansion chamber was measured to be in the 5 to $7 \times 10^{-5}$ mbar range and we fixed the position of the source with respect to the skimmer aperture to $1.7 \mathrm{~cm}$. We estimated the $\mathrm{NH}_{3}$ molecular beam impinging flux for each binary mixture by analyzing its partial pressure rise, $\left(P_{2}-P_{1}\right)$ in Figure 2a, corrected from the ionization cross section at the electron energy used in the mass spectrometer in the sample chamber. Combining the $\mathrm{NH}_{3}$ partial pressure rise, the pumping speed of the sample chamber and the designed molecular beam footprint on samples, we determined $\mathrm{NH}_{3}$ fluxes of $6.0 \pm 2.7 \times 10^{17}$ $\mathrm{NH}_{3} \cdot \mathrm{m}^{-2} \cdot \mathrm{s}^{-1}$ for $\mathrm{NH}_{3}$ seeded in $\mathrm{N}_{2}$ and $4.0 \pm 1.4 \times 10^{18} \mathrm{NH}_{3} \cdot \mathrm{m}^{-2} \cdot \mathrm{s}^{-1}$ for $\mathrm{NH}_{3}$ seeded in He. Flux uncertainties were calculated as the standard deviation of $\left(P_{2}-P_{1}\right)$ values from sticking probability experiments performed with samples at room temperature or above because liquid nitrogen cooling increases the sample chamber pumping speed by an unknown value. However, this variation of pumping speed is not an issue for the collimated molecular beam flux impinging the samples since the $\mathrm{NH}_{3}$ is solely defined by the source conditions in the expansion chamber, as described above. Note that $\mathrm{He}$ and $\mathrm{N}_{2}$ do not stick on our bare samples covered with natural impurities and the $\mathrm{H}_{2} \mathrm{O}$ contamination of the molecular beam is below $0.1 \%$ as checked by mass spectrometry.

\subsection{Sticking probability measurement}

We used the well-known method of King and Wells ${ }^{21}$ to measure the sticking probabilities of $\mathrm{NH}_{3}$ on $\mathrm{W}$ and $316 \mathrm{~L}-\mathrm{SS}$ at normal incidence. It consists in comparing in a single experiment the reflection of $\mathrm{NH}_{3}$ on the sample of interest with the reflection on a reference sample having unity reflection. It is thus a self-calibrated method giving absolute values. The reference sample, Flag in Figure 1, is made from an inert material that is quartz (Goodfellow) in the present study. The $\mathrm{NH}_{3}$ reflection is obtained by measuring the $\mathrm{NH}_{3}$ partial pressure with the mass spectrometer tuned on $m / z=17 a m u / e$. The mass spectrometer is located behind the sample such that the eventual 
anisotropy of the reflection is cancelled out by multiple reflection in the sample chamber. A typical sticking probability measurement is shown in Figure $2 \mathrm{a}$ and is explained in the following.

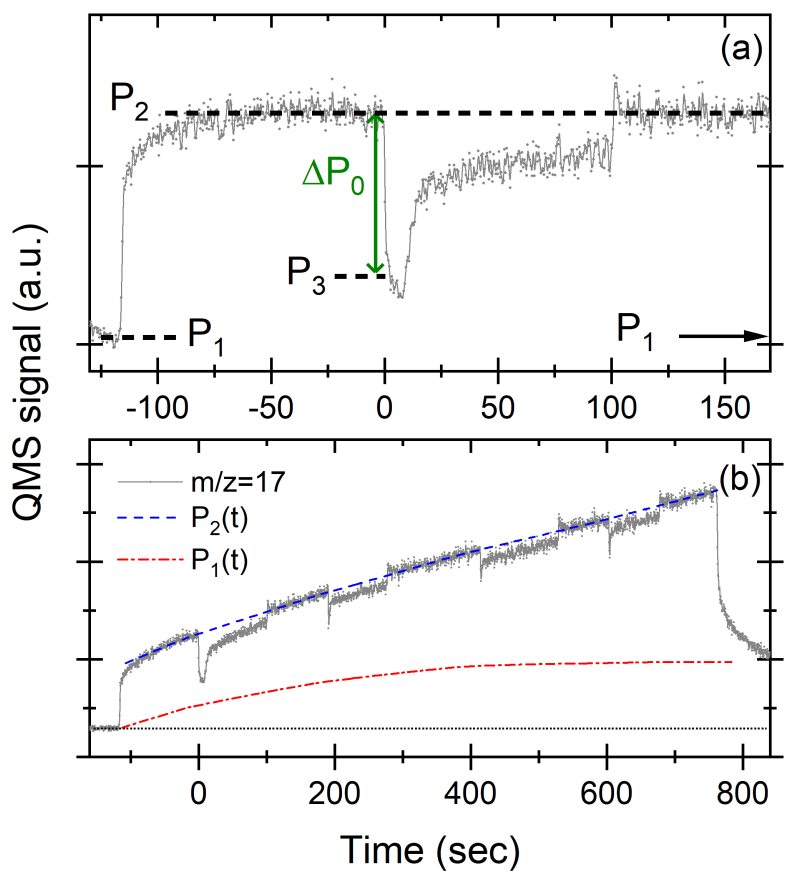

Figure 2: Sticking probability measurements obtained with $\mathrm{NH}_{3}$ at $E_{k}=55 \mathrm{meV}$ collimated on a W sample held at $220 \mathrm{~K}$. (a) corrected QMS signal according to the method described in section 2.3. (b) raw QMS signal at $m / z=17 \mathrm{amu} / e$. The dashed blue line shows the smooth function $P_{2}(t)$ reproducing the increase in partial pressure due to the adsorption/desorption of $\mathrm{NH}_{3}$ on the walls of the experimental setup. The dotted red line is $P_{1}(t)$, the downward-shifted and pressure rise-corrected $P_{2}(t)$ function, used to perform background subtraction leading to corrected QMS signals.

Initially, one measure the background partial pressure of $\mathrm{NH}_{3}$ in the sample chamber $\left(P_{1}\right)$. Then the collimated molecular beam is introduced in the sample chamber while the flag is positioned before the sample resulting in full reflection of the impinging molecules that reads as the pressure $P_{2}$. The difference $\left(P_{2}-P_{1}\right)$ is proportional to the impinging flux as discussed in the previous section. Next, the inert flag surface is removed and the $\mathrm{NH}_{3}$ beam collides with the sample which effectively pumps the molecular beam if its reflection probability is less than unity, i.e. there is a $\mathrm{NH}_{3}$ partial pressure drop to the value $P_{3}$. The initial sticking probability is defined as

$$
S_{0}=\frac{P_{2}-P_{3}}{P_{2}-P_{1}}
$$


Then, the $\mathrm{NH}_{3}$ partial pressure $P(t)$ will evolve back towards $P_{2}$ as the sample is covered with ammonia and the resulting time dependent sticking probability is simply defined as

$$
S(t)=\frac{P_{2}-P(t)}{P_{2}-P_{1}}
$$

Finally, once $P(t)$ has been recorded for a sufficient amount of time, the flag is raised before the sample to check that the impinging flux is constant and the molecular beam is stopped to check that the background pressure did not change significantly.

As it can be seen in Figure 2b, which corresponds to the raw (uncorrected) sticking probability measurement presented in Figure 2a, partial pressures $P_{1}$ and $P_{2}$ do change before and after the sticking probability measurement. There is a slow increase of partial pressures with time that last for about $\sim 1000 \mathrm{~s}$, indeed. A slow increase of partial pressure during sticking probability measurements is known to occur when the molecule of interest sticks on the wall of the sample chamber. Berenbak et al. have shown that sticking probability measurements performed for nitrogen monoxide in a stainless steel sample chamber lead to a similar behavior ${ }^{22}$. They provided a method to process such data based on the symmetry of the partial pressure evolution with time when the molecular beam is turned on and off. Therefore, we adopted a similar method to estimate the sticking probabilities from partial pressure traces. The pumping time constant of the sample chamber being about three orders of magnitude shorter than the time constant for the partial pressure evolution due to the sticking/desorption of $\mathrm{NH}_{3}$ from the wall, we obtain sharp partial pressure drop $\Delta P_{0}$ when the molecular beam strikes the sample (Figure 2a) that can be used to estimate the (initial) sticking probability in the limit of zero coverage $S_{0}{ }^{22}$. We use this sharp partial pressure drop to estimate the initial sticking probability with

$$
S_{0} \simeq \frac{\left|\Delta P_{0}\right|}{P_{2}(t)-P_{1}(t)}
$$

where $P_{2}(t)-P_{1}(t)$ is the estimate of the molecular beam flux-induced partial pressure rise. $P_{2}(t)$ is a smooth function that join the $\mathrm{NH}_{3}$ partial pressure measured while the molecular beam is in the 
sample chamber, from $\sim 100 \mathrm{~s}$ before dropping the flag until the molecular beam is removed from the sample chamber. $P_{1}(t)$ is the $P_{2}(t)$ function shifted downward and linearly transformed to join the $\mathrm{NH}_{3}$ partial pressure just before the introduction of the molecular beam in the sample chamber until $\sim 100 \mathrm{~s}$ after the molecular beam has been removed from the sample chamber (Figure $2 \mathrm{~b}$ ). Note that our method uses a symmetric combination in the spirit of Berenbak et al. method. Error bars on $S_{0}$ are obtained from a conservative estimate of the uncertainty of our method using $P_{1}(t)$ and $P_{2}(t)$ values at the ends of the time interval where sticking probability measurements are performed.

Thus, we estimate the time dependent sticking probability $S(t)$ with

$$
S(t) \simeq \frac{P_{2}(t)-P(t)}{P_{2}(t)-P_{1}(t)}
$$

where the initial sticking probability $S_{0}$ is obtained at $t_{0}\left(t_{0}=0 \mathrm{~s}\right.$ in Figure 2$)$.

The time integration of $S(t)$ in conditions of constant impinging flux $F$ is used to determine the evolution of the sticking probability with the $\mathrm{NH}_{3}$ coverage $\theta$ as follow

$$
S(\theta)=F \int_{t_{0}}^{t} S(t) d t
$$

\subsection{Samples preparation}

Two different samples were used in the present study: a polycrystalline tungsten (W) and a 316L stainless steel (316L-SS).

The W sample (99.99 wt.\% purity) was recrystallized in vacuum for $1 \mathrm{~h}$ at $1573 \mathrm{~K}$ by the manufacturer (A.L.M.T. Corp., Japan) and was subjected to mechanical and electro-polishing procedures at Aix-Marseille University. The W sample has a typical grain size of about $10 \mu \mathrm{m}$ and it is homogeneous throughout the bulk up to the surface as checked with scanning electron microscopy and transmission electron microscopy of cross-sectional laminae obtained by focused ion beam techniques $^{23}$. 
The 316L-SS sample was provided by Goodfellow as annealed and mechanically polished AISI 316L with the following specified content: $\mathrm{Cr} 18 \%$, Ni $10 \%$, Mo $3 \%, \mathrm{C}<0.03 \%$. According to the AISI standard, other chemical components in 316L-SS must be $\mathrm{Fe}>60 \%, \mathrm{Mn}<2.0 \%$, Si $<$ $1.0 \%, \mathrm{~N}<0.1 \%, \mathrm{P}<0.045 \%$ and $\mathrm{S}<0.03 \%$.

Before each $S_{0}$ and $S(\theta)$ measurements for a set of $\left(E_{k} ; T_{s}\right)$ parameters, samples were degassed to a temperature allowing desorption of adsorbed $\mathrm{NH}_{3}$ molecules but keeping any nitrogen fragment on the surface. In practice, the W sample was degassed at $1000 \mathrm{~K}$ while the 316L-SS sample degassing was limited to $700 \mathrm{~K}$ to limit the sample sublimation. AES analysis was realized on both samples before and after the sticking probability measurements to probe if the surface chemical composition evolved and if $\mathrm{NH}_{3}$ dissociation occurred. We note that the sticking probability measurements on 316L-SS initially evolved together with its surface composition. We present here results obtained on samples with stable surface composition.

\section{Results}

\subsection{Temperature dependence of $\mathrm{NH}_{3}$ initial sticking probability}

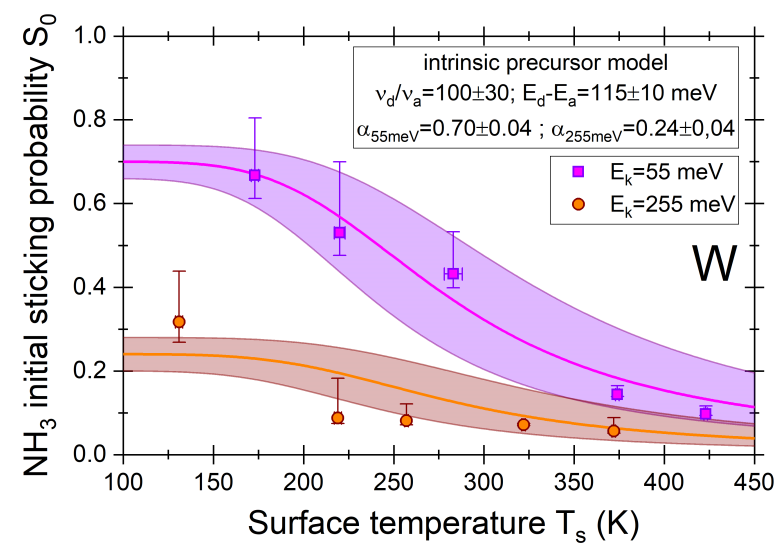

Figure 3: Initial sticking probability of ammonia on tungsten as a function of surface temperature for two different kinetic energies: $\mathrm{E}_{k}=55 \mathrm{meV}$ (magenta square) and $\mathrm{E}_{k}=255 \mathrm{meV}$ (orange circle). The curves are fit to the data employing the classic intrinsic precursor-mediated adsorption model (Equation 7) presented in the discussion section 4.1. 
Figure 3 presents the initial sticking probability $S_{0}$ of $\mathrm{NH}_{3}$ molecules impinging on a W sample for two different $E_{k}$ and as a function of $T_{s}$. It is found that $S_{0}$ are maximum at the lowest surface temperature. We have measured a maximum value of $S_{0}=0.67$ for $E_{k}=55 \mathrm{meV}$ and $T_{s}=173 \mathrm{~K}$. Note that large uncertainties on sticking probabilities are mostly present for $T_{s}<295 \mathrm{~K}$ because of liquid nitrogen cooling that induces larger $\mathrm{NH}_{3}$ partial pressure drifts (Figure 2b). For both investigated kinetic energies, $S_{0}$ decreases with the surface temperature. Nevertheless, the initial sticking probability $S_{0}$ remains at a quite high value above room temperature, with e.g. $S_{0}=0.10$ for $E_{k}=55 \mathrm{meV}$ and $T_{s}=423 \mathrm{~K}$. Finally, $S_{0}$ decreases when increasing $E_{k}$ with $S_{0}$ being divided by a factor of $\sim 3$ at $T_{s}=373 \mathrm{~K}$ when increasing $E_{k}$ from $55 \mathrm{meV}$ to $255 \mathrm{meV}$.

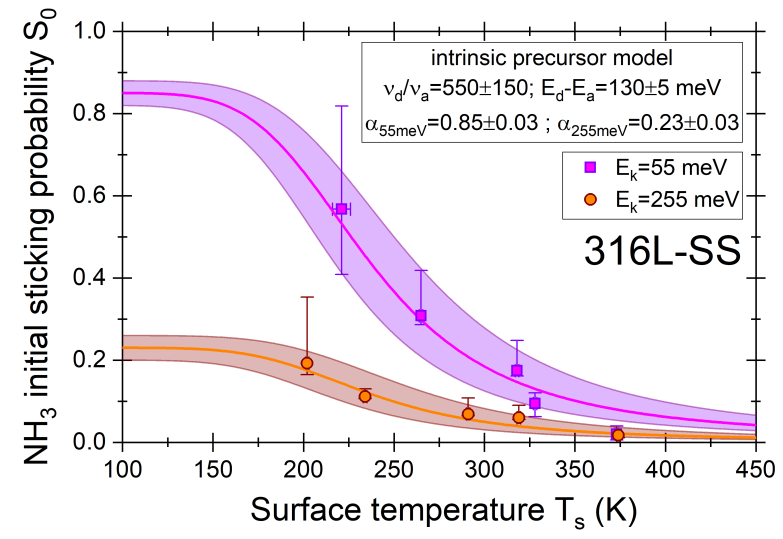

Figure 4: Initial sticking probability of ammonia on 316L stainless steel as a function of surface temperature for two $E_{k}$ : $55 \mathrm{meV}$ (magenta square) and $255 \mathrm{meV}$ (orange circle). The curves are fit to the data employing a classic intrinsic precursor-mediated adsorption model (Equation 7) presented in the discussion section 4.1 .

Figure 4 presents $S_{0}$ of $\mathrm{NH}_{3}$ molecules impinging a 316L-SS sample for two different $E_{k}$ and for various $T_{s}$. As in the case of $\mathrm{W}$, it is found that $S_{0}$ are maximum at the lowest surface temperature, with e.g. $S_{0}=0.57$ for $E_{k}=55 \mathrm{meV}$ and $T_{s}=221 \mathrm{~K} . S_{0}$ decreases with increasing $T_{s}$, reaching a value at the limit of detection of $S_{0}=0.02$ at $T_{s}=373 \mathrm{~K}$ and $E_{k}=55 \mathrm{meV}$. Finally, $S_{0}$ decreases with increasing the kinetic energy with, for example, $S_{0}$ being divided by a factor of $\sim 3$ at $T_{s}=318 \mathrm{~K}$ when increasing $E_{k}$ from $55 \mathrm{meV}$ to $255 \mathrm{meV}$.

$\mathrm{NH}_{3}$ sticking probabilities are rather in the same range for $\mathrm{W}$ and 316L-SS samples. However, sticking probabilities on $\mathrm{W}$ tend to be higher above room temperature with $S_{0}$ measurements being 
below the detection limit for $T_{s}>373 \mathrm{~K}$ on $316 \mathrm{~L}-\mathrm{SS}$ while it is still about $10 \%$ for $E_{k}=55 \mathrm{meV}$ on W.

\subsection{Coverage dependence of $\mathrm{NH}_{3}$ sticking probability}

Now, we focus on the influence of $\mathrm{NH}_{3}$ surface coverage on $\mathrm{NH}_{3}$ sticking probabilities. As described in section 2.3 and Figure 2b, the time evolution $S(t)$ for $\mathrm{NH}_{3}$ is recorded during a sticking probability measurement. Integrating the product of $S(t)$ with the constant beam flux (Equation 6) allows to determine the $\mathrm{NH}_{3}$ sticking probability dependence on the $\mathrm{NH}_{3}$ coverage $S(\theta)$. The result of this integration is shown in Figure 5 for $\mathrm{W}$ and in Figure 6 for 316L-SS, both in the case of $\mathrm{NH}_{3}$ impinging samples at $55 \mathrm{meV}$ with normal incidence. We limited the number of traces to three in both figures for the sake of clarity, the sticking probability behavior as a function of coverage being similar for both samples in the investigated temperature range.

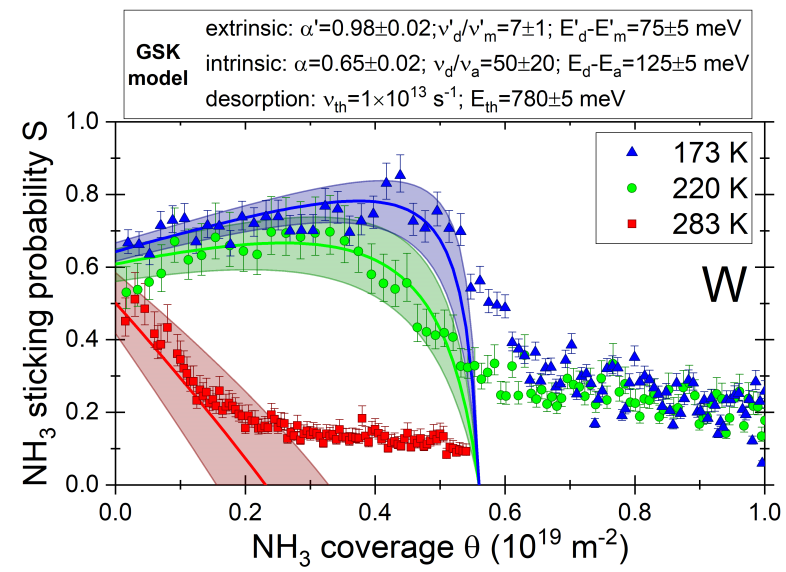

Figure 5: Sticking probability of $\mathrm{NH}_{3}$ as a function of ammonia coverage on tungsten. The kinetic energy of $\mathrm{NH}_{3}$ is $55 \mathrm{meV}$. The curves are a fit to the data employing the GSK precursors-mediated model (Equation 15) presented in the discussion section 4.2 with a saturation (monolayer) coverage of $0.56 \times 10^{19} \mathrm{~m}^{-2}$.

On both W and 316L-SS samples and for $T_{s}<265 \mathrm{~K}$, we found that the $\mathrm{NH}_{3}$ sticking probability is initially increasing as the $\mathrm{NH}_{3}$ surface coverage builds up until it reaches a maximum value. Then the sticking probability declines rather smoothly to an almost constant value which is different than zero, a so-called steady-state sticking. The maximum sticking probability is found 
to be $\sim 10 \%$ higher than $S_{0}$. The steady-state sticking can have probabilities in the $10-20 \%$ range up to $\mathrm{NH}_{3}$ coverage above $1.0 \times 10^{19} \mathrm{~m}^{-2}$. It becomes more difficult to discriminate $S_{0}$ from a maximum sticking probability as the surface temperature increases, as it can be seen in Figure 5 for $T_{s}=283 \mathrm{~K}$ and in Figure 6 for $T_{s}=265 \mathrm{~K}$. Thermal desorption of adsorbed $\mathrm{NH}_{3}$ from the surface is at the origin of this behavior as well as the cause of the reduction of the steady-state sticking when increasing the surface temperature (at $283 \mathrm{~K}$ in Figure 5 and at $265 \mathrm{~K}$ in Figure 6). These features will be examined in details in the discussion section 4 .

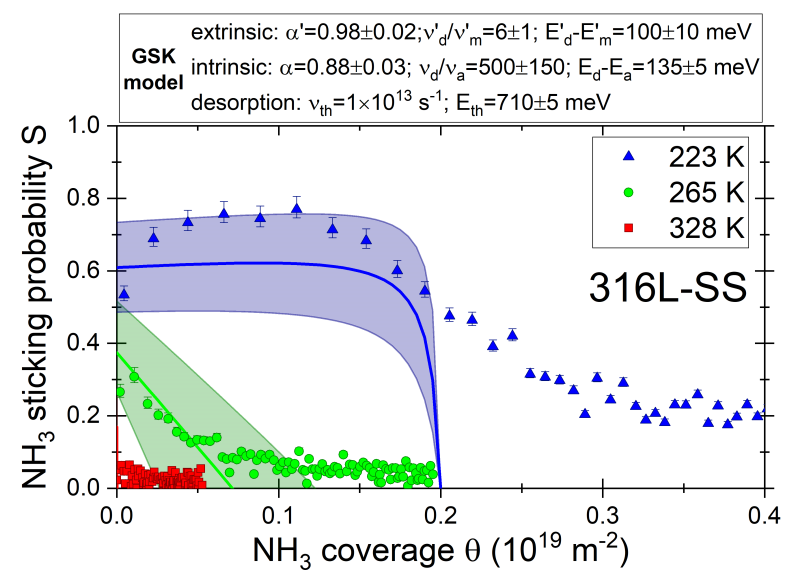

Figure 6: Sticking probability of $\mathrm{NH}_{3}$ as a function of ammonia coverage on 316L stainless steel. The kinetic energy of $\mathrm{NH}_{3}$ is $55 \mathrm{meV}$. The curves are a fit to the data employing the GSK precursors-mediated model (Equation 15) presented in the discussion section 4.2 with a saturation (monolayer) coverage of $0.2 \times 10^{19} \mathrm{~m}^{-2}$.

\subsection{Surface chemical composition}

Figure 7 presents the surface chemical composition of 316L-SS and W samples, as seen from Auger electron spectroscopy (AES) performed at room temperature, after the complete sticking probability measurements series. These AES spectra are identical to the ones obtained before the realization of the sticking probability measurements. Majority elements at the 316L-SS surface were chromium, iron, oxygen and carbon. The W sample surface exhibited mostly tungsten and carbon. After desorbing adsorbed $\mathrm{NH}_{3}$ while keeping samples temperature below nitrogen desorption, we found no clear traces of nitrogen (within the noise level) on both samples even after a 
cumulative $\mathrm{NH}_{3}$ fluence of $4 \times 10^{21} \mathrm{NH}_{3} \cdot \mathrm{m}^{-2}$. Considering that our AES system should be able to detect a $\sim 10 \%$ monolayer nitrogen coverage, these observations pose an upper limit of $1 \times 10^{-3}$ for the dissociation probability of $\mathrm{NH}_{3}$ on $\mathrm{W}$ and 316L-SS. Thus, the measured sticking probabilities correspond to a non-dissociative adsorption of $\mathrm{NH}_{3}$.

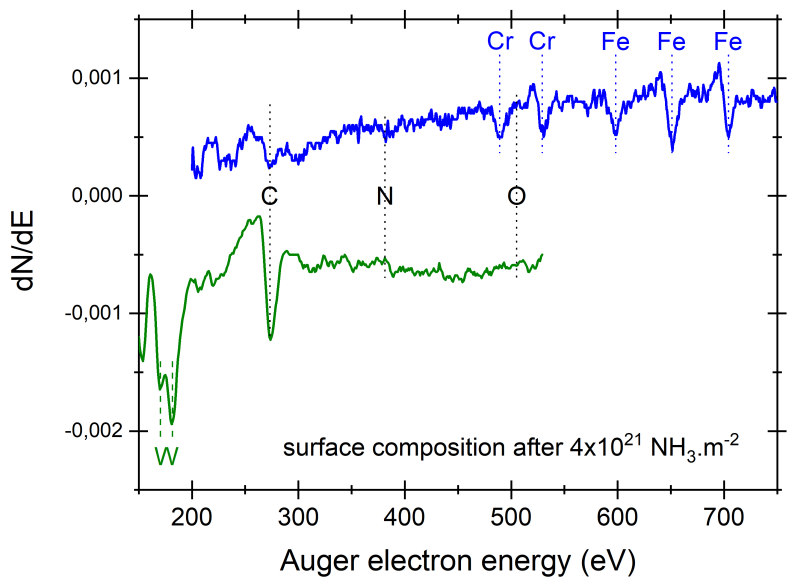

Figure 7: AES spectra of the 316L-SS sample (top blue spectra) and the W sample (bottom green spectra) at the end of the experimental campaign.

\section{Discussion}

\subsection{Initial sticking probability dependence with surface temperature: clas- sic intrinsic precursor-mediated adsorption}

The variation of a molecule initial sticking probability $S_{0}$ on a surface as a function of $E_{k}$ and $T_{s}$ reflects both kinetic and dynamical aspects of the adsorption process. In general, two types of adsorption mechanism are considered ${ }^{20}$ : the direct adsorption and the precursor-mediated adsorption. Direct adsorption occurs when incident molecules adsorb in a deep potential energy well directly upon impact with the surface. The main features of the direct adsorption mechanism is a weak dependency of $S_{0}$ on the surface temperature $T_{s}$ since the time allowed for energy exchange is restricted by the single impact condition. The dependence of $S_{0}$ on increasing the kinetic energy $E_{k}$ in direct adsorption depends on the presence of an activation energy for adsorption. In presence 
of an adsorption energy barrier, $S_{0}$ will increase with $E_{k}$ because of energy barrier activation. In absence of an adsorption energy barrier, $S_{0}$ will rather decrease with increasing $E_{k}$ because kinetic energy loss upon a single impact becomes insufficient to stay in the adsorption well. In contrast, precursor-mediated adsorption is the results of a two steps process, at least. Upon impact with the surface, incident molecules are first trapped in a shallow potential energy well where they equilibrate with the surface. Then, a kinetic competition between desorption from the shallow well and adsorption in a deeper potential well sets in. The main features of the precursor-mediated adsorption is a decrease of $S_{0}$ with increasing $E_{k}$ for a similar reason than in the case of barrier-less direct adsorption. However, a strong dependency of $S_{0}$ on increasing $T_{s}$ is expected for precursormediated adsorption because of the kinetic competition between desorption and adsorption which is in general thermally activated. $S_{0}$ can actually increase or decrease with increasing $T_{s}$ depending on the position of the activation energy barrier between the trapping well and the adsorption well with respect to the vacuum level.

Figure 3 and Figure 4 show that $S_{0}$ decreases when increasing $E_{k}$ as well as varies when increasing $T_{s}$. As described in the previous paragraph, these features are signatures of a precursormediated adsorption of $\mathrm{NH}_{3}$ on both tungsten and 316L stainless steel. Furthermore, the decrease of $S_{0}$ whith increasing $T_{s}$ indicates that the barrier for desorption from the shallow trapping well is higher than the barrier to go from the trapping well to the deeper adsorption well ${ }^{20}$. This conclusion is obtained from a simple kinetic model that considers solely a (intrinsic) precursor on the bare surface of the $\mathrm{NH}_{3}$ /surface system. In this model, impinging $\mathrm{NH}_{3}$ molecules undergo a first dynamical step, trapping in the precursor well with a probability $\alpha$. The second step is the kinetic competition between adsorption in a deeper well and desorption. Assuming that the surface density of $\mathrm{NH}_{3}$ precursors is small and constant and that rates follow thermal (Arrhenius) laws, the initial sticking probability can be expressed as

$$
S_{0}=\alpha\left(\frac{k_{a}}{k_{a}+k_{d}}\right)=\frac{\alpha}{1+\frac{v_{d}}{v_{a}} \exp \left(\frac{-\left(E_{d}-E_{a}\right)}{k_{b} T_{s}}\right)}
$$


where $\alpha$ is the trapping probability in the precursor well, $k_{a}$ and $k_{d}$ are the rate coefficients for, respectively, adsorption and desorption, $E_{a}$ and $E_{d}$ the corresponding activation energy barriers, $v_{a}$ and $v_{d}$ the corresponding attempt frequencies, $k_{b}$ the Boltzmann constant and $T_{s}$ the surface temperature.

In order to extract kinetic parameters defining the $T_{s}$ dependency of $S_{0}$ from present results, we performed a global fit of the experimental data at both investigated kinetic energies by Equation 7 with a least squares method. The results of the fitting procedure are shown by solid curves in Figure 3 and Figure 4. The fitting procedure gives quite similar results on both surfaces with $E_{d}-E_{a} \sim 125 \mathrm{meV}$ confirming that the barrier for desorption from the precursor well is higher than the barrier for adsorption as discussed above. Furthermore, $v_{d} / v_{a}>10$ is found on both surfaces, which suggests that reaching the adsorption barrier is more difficult than accessing the desorption barrier in configuration space. This simple kinetic model is able to reproduce the $S_{0}$ dependency on $\mathrm{T}_{s}$ (Figure 3 and Figure 4). However, such classic intrinsic precursor-mediated adsorption model predicts that the sticking probability should decrease linearly with coverage in contradiction with Figure 5 and Figure 6.

The observation of a sticking probability that does not decreases linearly with coverage was rationalized by Kisliuk in 1957 with a kinetic model that considers, in addition to the classic intrinsic precursor, an adsorbate-assisted adsorption through the so-called extrinsic precursor which is a $\mathrm{NH}_{3}$ precursor state existing on the $\mathrm{NH}_{3}$ partially covered surface. The Kisliuk model ${ }^{24}$ has been used successfully to explain a large range of gas/surface systems where $S(\theta)$ varies non-linearly with coverage, including sticking probability remaining constant with increasing adsorbate coverage. However, the Kisliuk model cannot explain an adsorbate sticking probability that increases with adsorbate coverage because an important initial assumption is the identical trapping probability for the intrinsic and extrinsic precursors. An increase of the sticking probability with coverage can be obtained if one assumes the absence of an intrinsic precursor and considers instead a direct adsorption together with an extrinsic precursor $^{25}$, the so-called modified Kisliuk model ${ }^{26}$. However, this modified Kisliuk model cannot explain an initial sticking probability that decreases with 
surface temperature since in the zero coverage limit only the direct adsorption term matters. Therefore, in order to explain our results one needs to generalize the Kisliuk model to the case where the trapping probabilities of the intrinsic and extrinsic precursors are different. This is the subject of the next section.

\subsection{Sticking probability dependence with surface temperature and $\mathrm{NH}_{3}$ sur- face coverage: generalization of the Kisliuk precursors-mediated model and analysis of simplifying hypotheses}

On the one hand, Figure 3 and Figure 4 showed that $S_{0}$ decreases with $T_{s}$ which cannot be explained by the combination of a direct adsorption and an extrinsic precursor (modified Kisliuk model). On the other, Figure 5 and Figure 6 presented an increase of $S(\theta)$ with coverage which cannot be reproduced by the combination of an intrinsic and extrinsic precursors with identical trapping probabilities (Kisliuk model). We derive in the following a generalization of the Kisliuk model where the trapping probabilities of the intrinsic and extrinsic precursors are different. Then we analyze several simplifying hypotheses. Finally, we isolate a Generalized and Separable Kisliuk (GSK) model that quantitatively reproduces our experimental results and readily separates the two precursors contribution to the sticking probability, easing the model interpretation.

Let us first consider the Kisliuk model (Figure 8 top) where the adsorbed $\mathrm{NH}_{3}$ noted $A(a)$ is defined on the bare surface, the intrinsic $\mathrm{NH}_{3}$ precursor $A^{*}$ is defined on the bare surface and the extrinsic $\mathrm{NH}_{3}$ precursor $A^{\prime}$ exists on the $\mathrm{NH}_{3}$ covered surface. The identical trapping probability in both precursor wells is termed $\alpha$ while their desorption rates are noted $k_{d}$ and $k_{d}^{\prime}$, respectively. Provision is made for a transfer between those two precursors population through kinetic rates $k_{m}$ and $k_{m}^{\prime}$ and the final adsorption step in the deep well $A(a)$ is realized through the intrinsic precursor with rate $k_{a}$. The Kisliuk model gives the evolution of the sticking probability with coverage, in terms of kinetic rates ${ }^{26}$, as

$$
S(\theta)=\frac{\alpha}{1+\left(\frac{\theta}{1-\theta}\right) K}\left(\frac{k_{a}}{k_{a}+k_{d}}\right)
$$


where $\theta$ is the normalized coverage, i.e. the coverage divided by the saturation (monolayer) coverage, and with

$$
K=\left(\frac{k_{d}^{\prime}}{k_{m}^{\prime}+k_{d}^{\prime}}\right)\left(\frac{k_{a}+k_{d}+k_{m}}{k_{a}+k_{d}}\right)
$$

which gives rise to a range of behavior (Figure 8 bottom) for different value of $K$. In this model, an increasing sticking probability with an increasing coverage cannot be obtained because $\alpha$ is the same for trapping in precursors $A^{*}$ and $A^{\prime}$.

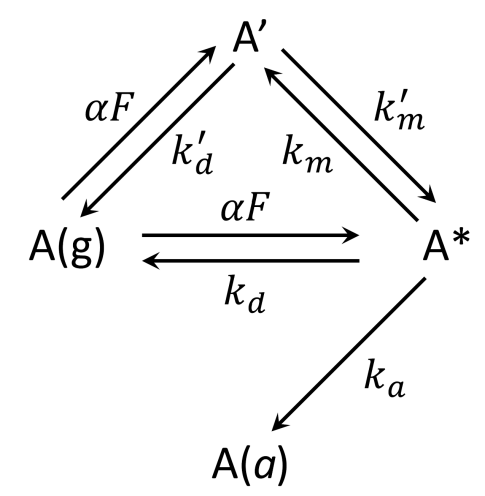

Kisliuk model

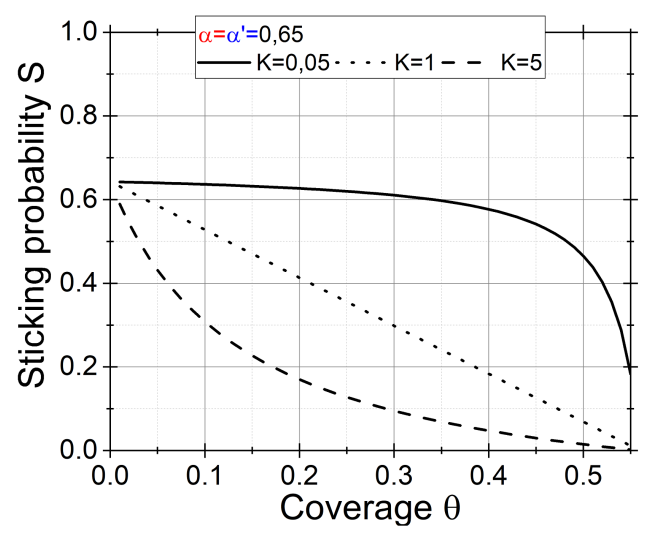

Figure 8: Top: Definition of the Kisliuk model. Bottom: illustration of the resulting sticking probability behaviour as a function of coverage for a set of three different $\mathrm{K}$ parameters.

We now derive a Generalized Kisliuk (GK) model (Figure 9) that allows to describe an increasing $S(\theta)$ by defining $\alpha$ and $\alpha^{\prime}$ the trapping probabilities in $A^{*}$ and $A^{\prime}$, respectively. We also include the thermal desorption of adsorbed $\mathrm{NH}_{3} A(a)$ with the kinetic rate $k_{t h}$. 

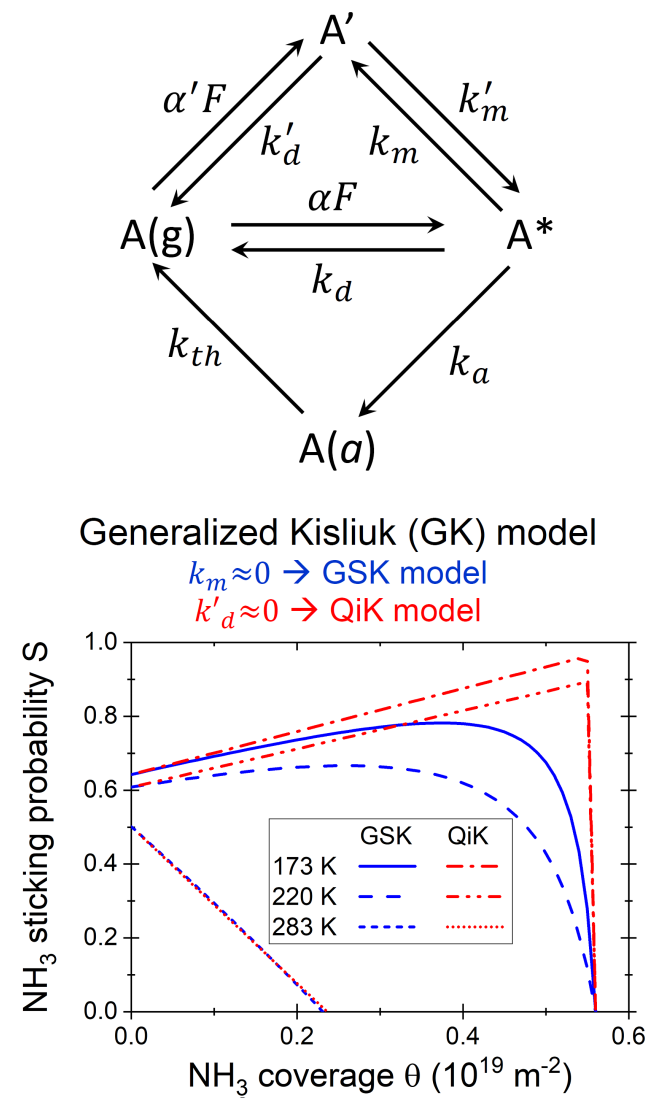

Figure 9: Top: Definition of the proposed Generalized Kisliuk (GK) model. Note that the trapping probability in the precursors are different i.e. $\alpha \neq \alpha^{\prime}$. Assuming $k_{m} \approx 0$ leads to the Generalized and Separable Kisliuk (GSK) model. Posing $k_{d}^{\prime} \approx 0$ leads to the Quasi-intrinsic Kisliuk (QiK) model. Bottom: comparison of the GSK and QiK modelling of the $\mathrm{W}$ experiments presented in Figure 5. 
The sticking probability of the gas phase species $A(g)$ impinging the surface can be defined as

$$
S(\theta)=\frac{1}{F}\left(k_{a}\left[A^{*}\right]-k_{t h}[A(a)]\right)
$$

where $[A(a)]=\theta$ is the normalized coverage of the adsorbed species and $F$ is the flux density of $A(g)$ divided by the saturation coverage.

Assuming a steady-state of small concentrations of precursors, one can derive the following Generalized Kisliuk (GK) model

$$
S^{G K}(\theta)=S_{\text {int }}^{G K}(\theta)+S_{\text {ext }}^{G K}(\theta)-\frac{k_{t h} \theta}{F}
$$

with

$$
S_{\text {int }}^{G K}(\theta)=\alpha(1-\theta)\left(\frac{k_{a}}{k_{a}+k_{d}+k_{m}\left(\frac{\theta K^{\prime}}{1-\theta+K^{\prime}}\right)}\right)
$$

and

$$
S_{\text {ext }}^{G K}(\theta)=\alpha^{\prime} \theta\left(\frac{k_{a}}{\left(k_{a}+k_{d}\right)\left(1+\left(\frac{1}{1-\theta}\right) K^{\prime}\right)+k_{m}\left(\frac{\theta}{1-\theta}\right) K^{\prime}}\right)
$$

where

$$
K^{\prime}=\frac{k_{d}^{\prime}}{k_{m}^{\prime}}
$$

The present GK model is a simplification of the generalized expression by Cassuto and King ${ }^{25}$ in that thermal desorption of the adsorbed species is not allowed to occur through the precursors. The GK model offers the flexibility for describing a wider range of sticking probability variation with coverage than the Kisliuk model but it does not offer a simple effective parameter $(K)$ like the Kisliuk model. However, $K$ is not readily reducible making temperature dependent data fitting complex with the Kisliuk model unless strong simplifying assumptions involving several kinetic rates are made. In contrast, the GK model car inform on the precursors kinetics, assuming that rates follow Arrhenius laws, thanks to its 8 independent free parameters $\left(\alpha, \alpha^{\prime}, v_{d} / v_{a}, v_{m} / v_{a}, v_{d}^{\prime} / v_{m}^{\prime}\right.$, $E_{d}-E_{a}, E_{m}-E_{a}$ and $\left.E_{d}^{\prime}-E_{m}^{\prime}\right)$. Nevertheless, these free parameters appear in both precursor terms 
(Equation 12 and 13) making experimental data fitting complicated. Therefore, we analyze in the following two choices of light simplifying hypotheses for the GK model, involving only a single kinetic rate, that will allow to reduce the number of free parameters as well as to ease the extraction of kinetic parameters for the precursors.

The first simplifying hypothesis assumes that an intrinsic precursor cannot become an extrinsic precursor, i.e. $k_{m} \approx 0$. In other words, we pose that if an intrinsic precursor $A^{*}$ on the bare surface jumps towards an adsorbate island $A(a)$, it would more likely desorb $(A(g))$ rather than become an extrinsic precursor $A^{\prime}$. This hypothesis allows to obtain a separable model since $S_{\text {int }}$ will now depend only on the intrinsic precursor kinetics. The so-called Generalized and Separable Kisliuk (GSK) model has the following simplified expression

$$
S^{G S K}(\theta)=S_{\text {int }}^{G S K}(\theta)+S_{\text {ext }}^{G S K}(\theta)-\frac{k_{t h} \theta}{F}
$$

where

$$
S_{i n t}^{G S K}(\theta)=\alpha(1-\theta)\left(\frac{k_{a}}{k_{a}+k_{d}}\right)
$$

and

$$
S_{\text {ext }}^{G S K}(\theta)=\frac{\alpha^{\prime} \theta}{1+\left(\frac{1}{1-\theta}\right) K^{\prime}}\left(\frac{k_{a}}{k_{a}+k_{d}}\right)
$$

with $K^{\prime}$ being the same as in the GK model (Equation 14).

The precursors kinetics in the GSK model is entirely defined by 6 free parameters, the intrinsic precursor term $S_{\text {int }}^{G S K}$ having only 3 free parameters $\left(\alpha, v_{d} / v_{a}\right.$, and $\left.E_{d}-E_{a}\right)$. Given the dependency of $S^{G S K}$ with adsorbate coverage $\theta$, it follows that the analysis of the (initial) sticking probability in the limit of zero coverage $S_{0}$ as a function of surface temperature (Figure 3 and Figure 4) determines the 3 kinetic parameters of the intrinsic precursor. Once determined, the 3 others kinetic parameters related to the extrinsic precursor are readily adjusted on the sticking probability $S(\theta)$ experiments as a function of surface temperature (Figure 5 and Figure 6).

The Generalized and Separable Kisliuk model is applied to our experimental dataset obtained 
for $\mathrm{NH}_{3}$ impinging on $\mathrm{W}$ (Figure 5) and 316L-SS (Figure 6). The GSK model (Equation 15) allows to reproduce the $S(\theta)$ behavior. In particular, the 6 free parameters defining the precursors kinetics are adjusted for $T_{s}<250 \mathrm{~K}$ as described in the previous paragraph. The inclusion and adjustment of the thermal desorption term $k_{t h} \theta / F$ being necessary for $T_{s}>250 \mathrm{~K}$.

We now turn to a second, different, simplifying hypothesis that can be applied to the Generalized Kisliuk model. One could argue that an extrinsic precursor is a way to change the trapping probability of the impinging species into an intrinsic precursor as a function of coverage, accounting for a better accommodation of the kinetic energy of the incident $\mathrm{NH}_{3}$ in presence of already adsorbed $\mathrm{NH}_{3}$ in our present case. This argument would translate in considering that the extrinsic precursor transfers totally to the intrinsic precursor, i.e. $k_{d}^{\prime} \approx 0$ that is desorption from the extrinsic precursor is negligible. This hypothesis reduces the GK model of Equation 11 to an expression related to the intrinsic precursor-mediated adsorption of Equation 7, the so-called Quasi-intrinsic Kisliuk model (QiK) in the following, which writes

$$
S^{Q i K}(\theta)=\left(\alpha(1-\theta)+\alpha^{\prime} \theta\right)\left(\frac{k_{a}}{k_{a}+k_{d}}\right)-\frac{k_{t h} \theta}{F}
$$

The precursor kinetics in the QiK model is defined by only 4 free parameters $\left(\alpha, \alpha^{\prime}, v_{d} / v_{a}\right.$, and $E_{d}-E_{a}$ ). Similarly to the GSK model, three of these precursor kinetic parameters can be determined by analysis of $S_{0}$ as a function of $\mathrm{T}_{s}$. The last precursor kinetic parameter $\left(\alpha^{\prime}\right)$ being determined by analysis of $S(\theta)$ measurements. The QiK model is applied to our experimental dataset obtained for $\mathrm{NH}_{3}$ impinging on $\mathrm{W}$ and compared to the results of the GSK modelling (Figure 9 bottom). Both QiK and GSK models are able to describe the increase of the sticking probability with $\mathrm{NH}_{3}$ coverage, which was lacking in the Kisliuk model. However, the QiK model exhibits a sharp drop of the sticking at completion of the saturation coverage, in contrast to the experimental observation of a smooth decline. Thus, the GSK model appears to be the best simplifying hypothesis to reproduce our experimental data. 


\subsection{Sticking probability dependence with surface temperature and $\mathrm{NH}_{3}$ sur- face coverage: dynamics and kinetics results from the GSK modelling}

Table 2 summarizes trapping probabilities, attempt frequencies ratios and activation energy barriers differences that have been used in order to reproduce our experiments with the Generalized and Separable Kisliuk model. Note that the fitting results of the intrinsic contribution in the GSK precursors-mediated model are identical (within uncertainties) to the ones found for the classic intrinsic precursor-mediated adsorption model, as it should be considering that Equation 15 reduces to Equation 7 in the limit of zero coverage.

Table 2: Parameters used in the GSK precursors-mediated adsorption models. $\alpha$ and $\alpha$ ' are for $E_{k}=55 \mathrm{meV}$. Energies are expressed in $\mathbf{m e V}$.

\begin{tabular}{lccc}
\hline \hline Kinetic step & Parameters & $\mathrm{W}$ & 316L-SS \\
\hline \multirow{3}{*}{ Extrinsic } & $\alpha$ & $0.98 \pm 0.02$ & $0.98 \pm 0.02$ \\
& $v_{d}^{\prime} / v_{m}^{\prime}$ & $7 \pm 1$ & $6 \pm 1$ \\
& $E_{d}^{\prime}-E_{m}^{\prime}$ & $75 \pm 5$ & $100 \pm 10$ \\
\hline \multirow{3}{*}{ Intrinsic } & $\alpha$ & $0.65 \pm 0.02$ & $0.88 \pm 0.03$ \\
& $v_{d} / v_{a}$ & $50 \pm 20$ & $500 \pm 150$ \\
& $E_{d}-E_{a}$ & $125 \pm 5$ & $135 \pm 5$ \\
\hline \multirow{2}{*}{ Desorption } & $v_{t h}$ & $1 \times 10^{13} \mathrm{~s}^{-1}$ & $1 \times 10^{13} \mathrm{~s}^{-1}$ \\
& $E_{t h}$ & $780 \pm 5$ & $710 \pm 5$ \\
\hline \hline
\end{tabular}

We find that the trapping probability of $\mathrm{NH}_{3}$ as an extrinsinc precursor is the same on both metals. This result is consistent with the similar nature of the extrinsic precursor, i.e. a $\mathrm{NH}_{3}$ molecule trapped on an island of adsorbed $\mathrm{NH}_{3}$ above a contaminated metal (Figure 7). Furthermore, the trapping probability of the extrinsic precursor for $E_{k}=55 \mathrm{meV}$ increases towards unity (0.98) on $\mathrm{W}$ and 316L-SS, which points out the optimal energy transfer expected during an elastic collision between a projectile and a target of identical mass. Finally, the kinetic parameters for the extrinsic precursor are quite similar for both metals, with $v_{d}^{\prime} / v_{m}^{\prime} \sim 7$ and $E_{d}^{\prime}-E_{m}^{\prime} \sim 85 \mathrm{meV}$, indicating that desorption is more accessible in configuration space than a transfer to the intrinsic precursor.

Regarding the trapping probability of $\mathrm{NH}_{3}$ as an intrinsic precursor, it is found higher on 316LSS (0.88) than on $\mathrm{W}(0.65)$. The lighter elements composing 316L-SS presumably enhance the 
energy transfer necessary for trapping of $\mathrm{NH}_{3}$ as compared to $\mathrm{W}$. The kinetic parameters of the intrinsic precursor are markedly different for the two metals. In particular, $v_{d} / v_{a}$ is about 10 times higher on 316L-SS than on W indicating that the transfer of the intrinsic precursor to the adsorbed state is more difficult on $316 \mathrm{~L}-\mathrm{SS}$. We postulate that this is the results of the chemical heterogeneity of the 316L-SS alloy. Adsorption might preferably happen in the close environment of one chemical element ( $\mathrm{Cr}$ or Fe, see Figure 7) at the surface. This would be consistent with the observation that the $\mathrm{NH}_{3}$ saturation coverage, at which $S(\theta)$ declines, is smaller on $316 \mathrm{~L}-\mathrm{SS}\left(0.2 \times 10^{19}\right.$ $\left.\mathrm{NH}_{3} \cdot \mathrm{m}^{-2}\right)$ than on $\mathrm{W}\left(0.56 \times 10^{19} \mathrm{NH}_{3} \cdot \mathrm{m}^{-2}\right)$. It would be also consistent with the recent work of Yaala et al. ${ }^{19}$ where XPS showed that $\mathrm{NH}_{3}$ binds preferentially to $\mathrm{Cr}$ on a stainless steel surface. We note that the $\mathrm{NH}_{3}$ saturation coverage on $\mathrm{W}$ is rather high if one considers that the presence of an intrinsic precursor implies a relatively high density of weakly binding impurities that scatter impinging $\mathrm{NH}_{3}$. However, we stress that the root-mean-square roughness $\left(R_{q}\right.$, measured by AFM on $40 \times 40 \mu \mathrm{m}^{2}$ images) within grains of the present polycrystalline $\mathrm{W}$ is 3 times higher than the $R_{q}$ of a typical single crystal used for "clean" surface science and we think this is the reason for the observed relatively high $\mathrm{NH}_{3}$ saturation coverage.

Finally, assuming an attempt frequency of $10^{13} \mathrm{~s}^{-1}$ we found that the desorption barrier for adsorbed $\mathrm{NH}_{3}$ is higher on $\mathrm{W}(0.78 \mathrm{eV})$ than on $316 \mathrm{~L}-\mathrm{SS}(0.71 \mathrm{eV})$. Thanks to the separability of the GSK modelling, one can interpret $S_{0}$ and $S(\theta)$ dependency with surface temperature from sample to sample quite simply. Equations 16 and 17 shows that in the limit of zero coverage, $S_{0}=f\left(T_{s}\right)$ is solely ruled by the intrinsic precursor kinetics indeed. Thus the fact that $S_{0}=f\left(T_{s}\right)$ decreases more rapidly for $316 \mathrm{~L}-\mathrm{SS}$ than for $\mathrm{W}$ can be related to the larger prefactors ratio for 316L-SS which favors desorption from the intrinsic precursor. Comparing $S(\theta)=f\left(T_{s}\right)$ for W and 316L-SS, we could be able to discuss details of the extrinsic precursor kinetics knowing the intrinsic precursor kinetics. However, the lack of experimental data between $223 \mathrm{~K}$ and $265 \mathrm{~K}$ on both surfaces prevents a detailed comparison. We can simply note that similar kinetic parameters for the extrinsic precursor (Table 2) does not impede to reproduce the W and 316L-SS data. Future studies on W and 316L-SS should look in more details the 200 - $300 \mathrm{~K}$ surface temperature range. 
The use of single crystal $\mathrm{W}$ as well as model stainless steel surfaces is encouraged in order to define more precisely the impurities nature and their arrangement on the surface. This should help to define more precisely the various $\mathrm{NH}_{3}$ states deduced from the GSK modelling that we will discuss in the next section.

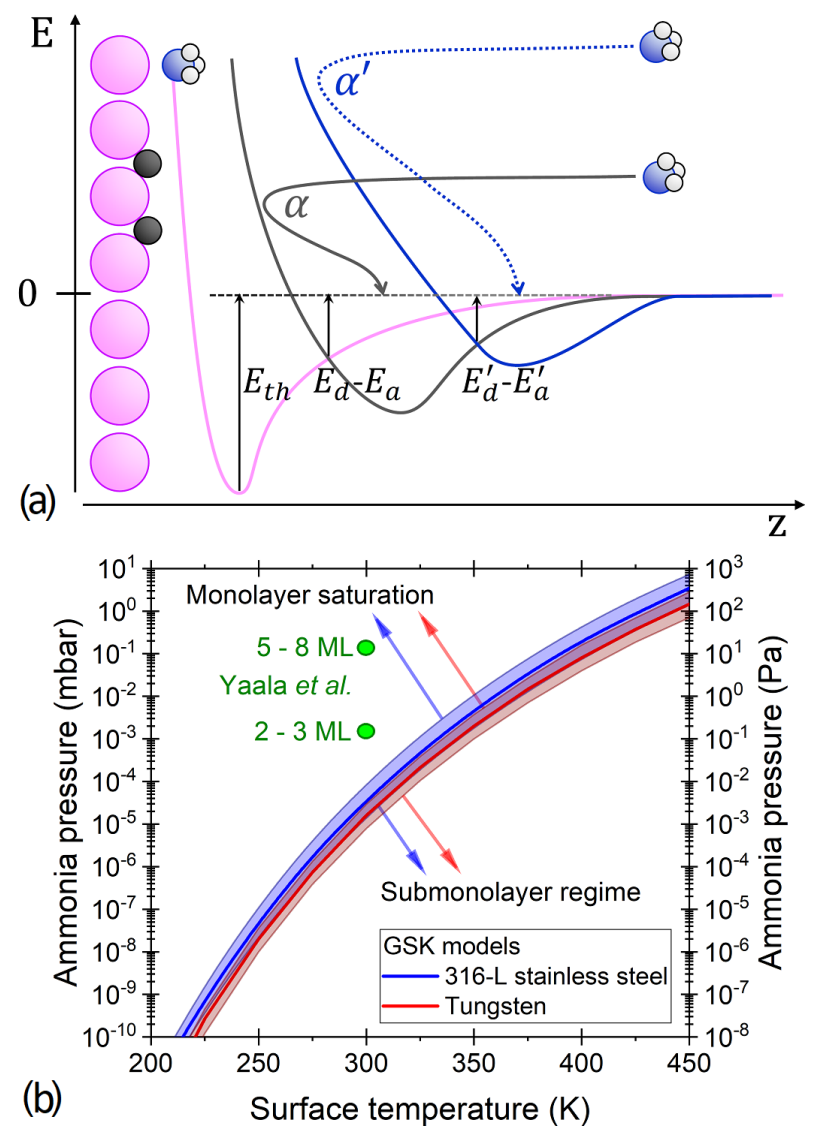

Figure 10: (a) Schematics of the energy landscape experienced by a $\mathrm{NH}_{3}$ molecule sticking on a technologically relevant and partially $\mathrm{NH}_{3}$ covered surface (W or 316L-SS ) within the GSK precursors-mediated adsorption model. The impinging $\mathrm{NH}_{3}$ can collide an adsorbed $\mathrm{NH}_{3}$ and traps with probability $\alpha^{\prime}$ in the extrinsic precursor well (blue). Or an impinging $\mathrm{NH}_{3}$ can collide near an impurity such as carbon and traps with probability $\alpha$ in the intrinsic precursor well (grey). Final adsorption of $\mathrm{NH}_{3}$ happens on clean or oxidized tungsten parts (pink). (b) GSK modelling of the $\mathrm{NH}_{3}$ monolayer (ML) completion in condition of static pressure as a function of surface temperature for $\mathrm{W}\left(1 \mathrm{ML}=0.56 \times 10^{19} \mathrm{NH}_{3} \cdot \mathrm{m}^{-2}\right)$ and $316 \mathrm{~L}-\mathrm{SS}\left(1 \mathrm{ML}=0.2 \times 10^{19} \mathrm{NH}_{3} \cdot \mathrm{m}^{-2}\right)$. Green dots are quartz micro-balance measurements from Yaala et al. ${ }^{19}$ with $1 \mathrm{ML}=0.62 \times 10^{19} \mathrm{NH}_{3} \cdot \mathrm{m}^{-2}$.

The overall results of the GSK modelling of $\mathrm{NH}_{3}$ sticking on tungsten and 316L stainless steel can be summarized in the simple one-dimensional energy profile along the adsorption path proposed in Figure 10a. Three potential energy wells are used to describe the sticking of $\mathrm{NH}_{3}$ on $\mathrm{W}$ 
and $316 \mathrm{~L}-\mathrm{SS}$, from bare surfaces up to the saturation of a monolayer, and all these wells represent non-dissociative adsorption state of ammonia. Access to these adsorption states depends on the initial impact site of the impinging $\mathrm{NH}_{3}$. If $\mathrm{NH}_{3}$ impinges on an already adsorbed $\mathrm{NH}_{3}$ it will trap with probability $\alpha^{\prime}$ in the extrinsic precursor well. If $\mathrm{NH}_{3}$ impinges on a weakly binding impurities, such as carbon, it will trap with probability $\alpha$ in the intrinsic precursor well. In this model, direct adsorption on clean parts of the surface is neglected because of the measured negative kinetic energy dependency (Figure 3 and Figure 4) and presumably because of the large mass mismatch between $\mathrm{NH}_{3}$ and the metals atom. The two energy barriers between the three adsorption wells were found below the vacuum level and the depth of the well closest to the surface $\left(E_{t h}\right)$ has been determined (Table 2). The non-dissociative states used in our model are consistent with the present AES surface chemical analysis where it is found that nitrogen atom density is negligible at both W and 316L-SS surfaces after a cumulative ammonia exposure of $4 \times 10^{21} \mathrm{NH}_{3} \cdot \mathrm{m}^{-2}$ (Figure 7). It is also consistent with Grunze et al. who showed that submonolayer coverage of an impurity such as oxygen reduces the dissociative rate of $\mathrm{NH}_{3}$ as well as stabilized the molecular adsorption of intact $\mathrm{NH}_{3}{ }^{11}$. The $E_{t h}$ obtained from our model indicates a desorption of $\mathrm{NH}_{3}$ around room temperature for submonoloyer coverage on $\mathrm{W}$ and $316 \mathrm{~L}-\mathrm{SS}$ samples. We note that similar desorption temperature were observed on clean single crystals ${ }^{14,15}$ and polycrystalline ${ }^{13}$ tungsten as seen with temperature programmed desorption.

The GSK modelling allows to estimate in which conditions saturation of the $\mathrm{NH}_{3}$ monolayer should be achieved and thus when $\mathrm{NH}_{3}$ multi-layer adsorption may occur. Applying the kinetic parameters from Table 2, we calculated at which static partial pressure of $\mathrm{NH}_{3}$ the monolayer coverage should be reached at a given temperature. Figure $10 \mathrm{~b}$ presents the results of this calculation for $\mathrm{W}$ and $316 \mathrm{~L}-\mathrm{SS}$. We found that at a surface temperature of $300 \mathrm{~K}, \mathrm{NH}_{3}$ monolayer saturation should happen at a pressure of $2 \times 10^{-5}$ mbar for $\mathrm{W}$ and $3 \times 10^{-5}$ mbar for $316 \mathrm{~L}-\mathrm{SS}$. These estimations are again consistent with the results of Yaala et al. ${ }^{19}$ who found 2 to 3 monolayers of $\mathrm{NH}_{3}$ on tungsten and stainless steel at a static $\mathrm{NH}_{3}$ pressure of $1 \times 10^{-3}$ mbar. 


\subsection{Beyond the GSK model: desorption spike, steady-state sticking and multi-layer adsorption}

The GSK model introduced in the previous section allowed to reproduce the sticking probability dependence of $\mathrm{NH}_{3}$ as a function of $\mathrm{NH}_{3}$ coverage and surface temperature for both metal surfaces. However, by construction the GSK model cannot describe the non-zero sticking probability found after saturation coverage, i.e. above $0.56 \times 10^{19} \mathrm{NH}_{3} \cdot \mathrm{m}^{-2}$ for $\mathrm{W}$ (Figure 5) and above $0.2 \times 10^{19}$ $\mathrm{NH}_{3} \cdot \mathrm{m}^{-2}$ for $316 \mathrm{~L}-\mathrm{SS}$ (Figure 6). Neither the GSK model can describe the desorption spike that occurs when molecular beam exposure of the surface is stopped (Figure 2).

First, we address the desorption spike, coming back to the typical sticking probability measurement procedure employed in this study. In Figure 2a the sticking probability is initiated at $t=0$ $\mathrm{s}$ and terminated at $t=100 \mathrm{~s}$. At the moment where the molecular beam exposure is stopped, we observed a desorption spike with a short decreasing tail towards $P_{2}(t)$. At $t=200 \mathrm{~s}, t=400 \mathrm{~s}$ and $t=600 \mathrm{~s}$, we repeated the sticking probability measurement (Figure $2 \mathrm{~b}$ ) and found systematically a pressure drop smaller than at $t=0 \mathrm{~s}$, indicating that these sticking probabilities are smaller than $S_{0}$. At $t=300 \mathrm{~s}, t=500 \mathrm{~s}$ and $t=700 \mathrm{~s}$, we repeatedly stopped the molecular beam exposure and observed the same desorption spike. Finally, the desorption spike amplitude taken from the pressure level before the molecular beam is stopped is found to be similar to the pressure drop amplitude observed for subsequent sticking measurement results. These similar desorption spike/pressure drop amplitudes can be explained by the combination of two mechanisms. A first part of the desorption spike/pressure drop amplitude comes from the steady-state sticking that develops in the first $30 \mathrm{~s}$ of molecular beam exposure and then within $3 \mathrm{~s}$ of repeated molecular beam exposure. We think that this steady state sticking is related to a multi-layer adsorption and will be discussed in the next paragraph. We rationalize the second part of the desorption spike/pressure drop amplitude as the manifestation of weakly bound $\mathrm{NH}_{3}$. These weakly bound states are transiently populated under the equilibrium condition of the molecular beam flux exposure, thus the initial sharp drop that superimposes to the steady-state sticking. Then weakly bound states are released once the molecular beam is stopped, leading to a desorption spike on-top of the return to the $P_{2}(t)$ level. The origin of 
these weakly bound $\mathrm{NH}_{3}$ could be at least two folds. First, the presence of an intrinsic precursor points to a relatively high density of weakly binding impurities, which could be carbon atoms that are found on both samples (Figure 7). Second, the saturation (monolayer) coverage of $\mathrm{NH}_{3}$ on several metals (Pt, Ni, W) was found by XPS to be independent of the metal or its crystallographic orientation, but rather determined by the repulsive (dipole-dipole) lateral interaction of $\mathrm{NH}_{3}$ in the first adsorption layer ${ }^{27}$ limiting its density to ca. $0.4 \times 10^{19} \mathrm{~m}^{-2}$. Thus the desorption spikes could originate from $\mathrm{NH}_{3}$ islands in the first adsorption layer that are densely packed upon molecular beam exposure.

Second, we turn to the steady-state sticking observation. As seen in Figure $2 b$, after the first $30 \mathrm{~s}$ of molecular beam exposure a steady-state sticking is measured, typically for hundreds of seconds without any sign of the sticking probability going to zero. This behavior indicates that the $\mathrm{NH}_{3}$ surface coverage does not saturate, Figure $2 \mathrm{~b}$ indicating that $\mathrm{NH}_{3}$ coverage of at least twice the "saturation" coverage has been reached. This observation should be the manifestation of the growth of $\mathrm{NH}_{3}$ multi-layer, as it has been measured recently by Yaala et al. ${ }^{19}$ for static $\mathrm{NH}_{3}$ pressure above $10^{-3}$ mbar. $\mathrm{NH}_{3}$ multi-layer growth was found also on $\mathrm{Pt}$, $\mathrm{Ni}$ and $\mathrm{W}$ surfaces below room temperature and at $10^{-10}$ mbar $^{27}$. On those surfaces, UPS measurement of the $\mathrm{NH}_{3}$ valence orbitals indicated that $\mathrm{NH}_{3}$ in the second layer is barely interacting with the metals and thus $\mathrm{NH}_{3}$ multi-layer condensation on the metals occurs. Figure 11 presents the evolution of the steady-state sticking $\left(S_{s s}\right)$ as a function of sample temperature for W. It is found that $S_{s s}$ decreases monotonously with increasing $T_{s}$ and $S_{s s}$ can have a probability of 0.10 for $E_{k}=55 \mathrm{meV}$ at $T_{s}=283$ $\mathrm{K}$. Note that at room temperature, $\mathrm{NH}_{3}$ desorption sets in on $\mathrm{W}$, as it is illustrated in Figure 5 at $283 \mathrm{~K}$, and a steady-state sticking is found even though saturation coverage is not reached. This observation would be consistent with a multi-layer growth occurring on adsorbed $\mathrm{NH}_{3}$ islands in an incomplete first-layer. Actually, Grunze et al. found on $\mathrm{Ni}^{27}$ that $\mathrm{NH}_{3}$ multi-layers islands could grow even though the first layer did not reach saturation, thanks to the repulsive dipole-dipole interaction in the first layer.

We are able to model the evolution of the steady-state sticking $S_{s s}$ as a function of surface 


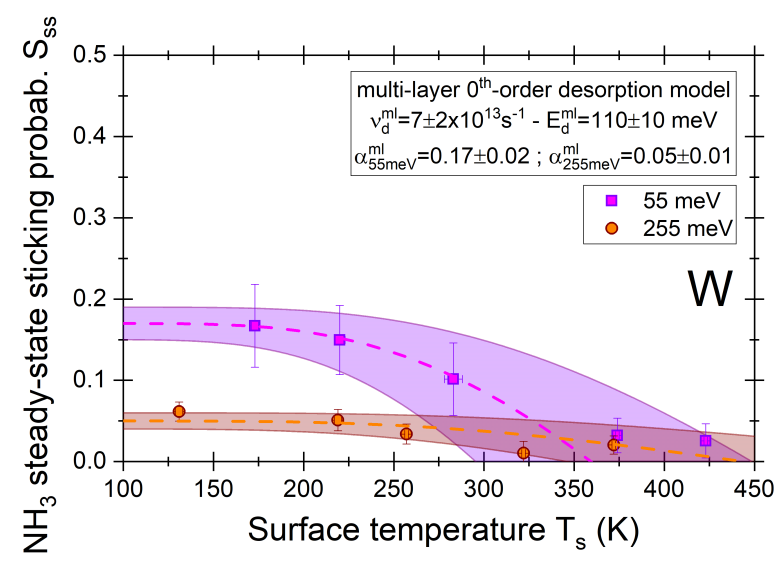

Figure 11: Steady-state sticking probability of ammonia on tungsten as a function of surface temperature for two different kinetic energies with different absolute flux. The $E_{k}=55 \mathrm{meV}$ beam has a flux of $1.2 \times 10^{13} \mathrm{NH}_{3} \mathrm{~s}^{-1}$ while the $E_{k}=255 \mathrm{meV}$ beam has a flux of $7.9 \times 10^{13} \mathrm{NH}_{3} \mathrm{~s}^{-1}$. The curves are fit to the data employing a model of zeroth order desorption from the multilayer (Equation 19).

temperature, with our molecular beam fluxes. Considering a zeroth order desorption of $\mathrm{NH}_{3}$ in the second layer, i.e. a desorption from the edge of $\mathrm{NH}_{3}$ islands in this second layer, one can describe the steady-state sticking with

$$
S_{s s}=\frac{1}{F}\left(\alpha^{m l} F-k_{d}^{m l}\right)
$$

and

$$
k_{d}^{m l}=v_{d}^{m l} \exp \left(-\frac{E_{d}^{m l}}{k_{b} T_{s}}\right)
$$

where $\alpha^{m l}$ is the trapping probability of an impinging $\mathrm{NH}_{3}$ on a $\mathrm{NH}_{3}$ multi-layer island, $F$ is the absolute molecular beam flux, $k_{d}^{m l}$ is the zeroth order desorption of $\mathrm{NH}_{3}$ in the ammonia multilayer, which temperature dependency is expressed in terms of the Arrhenius parameters $v_{d}^{m l}$ and $E_{d}^{m l}$. Figure 11 shows that this simple model is able to reproduce quite well the experimental dependency of the steady-state sicking with surface temperature for $\mathrm{W}$ with an attempt frequency on the order of $10^{13} \mathrm{~s}^{-1}$ and a desorption energy of about $110 \mathrm{meV}$. The $\mathrm{NH}_{3}$ trapping probability in the multi-layer $\alpha^{m l}$ is about 0.2 for $E_{k}=55 \mathrm{meV}$. We note that this is much smaller than the trapping probability in the extrinsic precursor $\alpha^{\prime}$ which was about unity. However, the extrinsic precursor in all Kisliuk models is defined only at a low density on the surface, i.e. impinging $\mathrm{NH}_{3}$ 
collides with $\mathrm{NH}_{3}$ adsorbed on a metal which has a desorption energy of about $700 \mathrm{meV}$ (Table 2). At a larger $\mathrm{NH}_{3}$ coverage in the $2^{\text {nd }}$ layer, typical for multi-layer adsorption, impinging $\mathrm{NH}_{3}$ will collide with $\mathrm{NH}_{3}$ in the $2^{\text {nd }}$ layer which has a lower desorption energy, estimated at about $110 \mathrm{meV}$ in Figure 11, i.e. on par with the impinging kinetic energy. Thus impinging $\mathrm{NH}_{3}$ would likely induce desorption of $\mathrm{NH}_{3}$ in the $2^{\text {nd }}$ layer explaining the overall lower trapping probability in the $2^{\text {nd }}$ layer $\left(\alpha^{m l}\right)$ than in the extrinsic precursor $\left(\alpha^{\prime}\right)$.

It would tempting to assume that $k_{d}^{m l} \approx k_{d}^{\prime}$ (thus $v_{d}^{m l} \approx v_{d}^{\prime}$ and $E_{d}^{m l} \approx E_{d}^{\prime}$ ) i.e. the desorption rate of ammonia in the second layer is similar to the desorption rate of the extrinsic precursor. However, as we just underlined, the local environment of $\mathrm{NH}_{3}$ as an extrinsic precursor should be different that the local environment of $\mathrm{NH}_{3}$ as part of a second layer. Considering that dipoledipole interaction should be repulsive within a $\mathrm{NH}_{3}$ layer, we can at least indicate a lower limit to $E_{d}^{\prime} \geq E_{d}^{m l}$ which is consistent with the values of $E_{d}^{\prime}-E_{m}^{\prime}$ found with the GSK modelling.

\section{Conclusions}

The present study combined supersonic molecular beam, mass spectrometry and surface science techniques to evidence the precursors-assisted dynamics and kinetics of $\mathrm{NH}_{3}$ adsorption on $\mathrm{W}$ and 316L-SS containing natural impurities. Experimental measurements of $\mathrm{NH}_{3}$ sticking probability give similar trends on both metals. The initial sticking probability $S_{0}$ decreases with the surface temperature $\mathrm{T}_{s}$ when approaching room temperature. The evolution of the sticking probability with $\mathrm{NH}_{3}$ surface coverage exhibits an initial increase followed by a rapid decline presumably when reaching saturation coverage. The sticking probability does not necessarily decline to zero since a steady-state sticking with probabilities above $10 \%$ was measured at room temperature and below. These features of the $\mathrm{NH}_{3}$ sticking probability, except for the steady-state sticking, are well reproduced for both surfaces with the Generalized and Separable Kisliuk model introduced in the present work. For both metals, the GSK model indicates that $\mathrm{NH}_{3}$ trapping probability is higher on $\mathrm{NH}_{3}$ partially covered surfaces (extrinsic precursor) than on bare surfaces (intrinsic precursor). 
Additionally, the desorption energy barriers from the two precursors wells are found higher than the energy barriers to transfer to an adsorption state closer to the surface. The analysis of the surface temperature dependency of the initial sticking probability $S_{0}$ and of the sticking probability $S(\theta)$ through the GSK model allows to inform on the intrinsic and extrinsic precursor kinetics separately in a two step process. This way we rationalize the decrease of the sticking probability of 3161-SS at a lower temperature than for $\mathrm{W}$ as the manifestation of its higher prefactors ratio in the intrinsic precursor which favors its desorption. We proposed that carbon contamination maybe at the origin of the intrinsic precursor kinetics, however, systematic studies on model surfaces of naturally contaminated W and 316L-SS are called upon in order to better characterize the origin of the observed behavior. We suggest that the GSK framework could be useful to further interpret the kinetics of other systems where both intrinsic and extrinsic precursors have been proposed, such as $\mathrm{O}_{2}$ on $\mathrm{Pt}^{28-31}$. Finally, the $\mathrm{NH}_{3}$ steady-state sticking was found to be related with multi-layer adsorption, consistent with the literature including spectroscopic measurements ${ }^{11,27}$ and quartz micro-balance measurements ${ }^{19}$. The kinetic parameters associated to the present GSK modelling is used to estimate at which $\mathrm{NH}_{3}$ static pressure multi-layer adsorption should starts on $\mathrm{W}$ and 316L-SS in a range of surface temperature of interest for ITER. These results are an important step for the predictive determination of the tritium inventory at the in-vessel and exhaust piping in the ITER experiment. This new understanding should help to assess nuclear safety and tritium recycling efficiency related to tritiated ammonia in realistic operational conditions.

\section{Acknowledgement}

The project leading to this publication has received funding from Excellence Initiative of AixMarseille University - A*MIDEX, a French Investissements d'Avenir programme as well as from the French National Research Agency (Grant No. ANR-18-CE05-0012). We thank the financial support of the French Federation for Magnetic Fusion Studies (FR-FCM). The views and opinions expressed herein do not necessarily reflect those of the ITER Organization. ITER is the Nuclear 
Facility INB no. 174. This paper applies new physics analysis related to tritiated ammonia formation which is not yet incorporated into the ITER technical baseline. The nuclear operator is not constrained by the results presented here.

\section{References}

(1) Ertl, G. Reactions at Surfaces: From Atoms to Complexity (Nobel Lecture). Angew. Chem., Int. Ed. 2008, 47, 3524-3535.

(2) Smil, V. Detonator of the population explosion. Nature 1999, 400, 415.

(3) Ye, L.; Nayak-Luke, R.; Bañares-Alcántara, R.; Tsang, E. Reaction: Green Ammonia Production. Chem 2017, 3, 712-714.

(4) Kallenbach, A.; Dux, R.; Fuchs, J. C.; Fischer, R.; Geiger, B.; Giannone, L.; Herrmann, A.; Lunt, T.; Mertens, V.; McDermott, R. et al. Divertor power load feedback with nitrogen seeding in ASDEX Upgrade. Plasma Phys. Controlled Fusion 2010, 52, 055002.

(5) Giroud, C.; Maddison, G. P.; Jachmich, S.; Rimini, F.; Beurskens, M. N. A.; Balboa, I.; Brezinsek, S.; Coelho, R.; Coenen, J. W.; Frassinetti, L. et al. Impact of nitrogen seeding on confinement and power load control of a high-triangularity JET ELMy H-mode plasma with a metal wall. Nucl. Fusion 2013, 53, 113025.

(6) Gruber, O.; Sips, A. C. C.; Dux, R.; Eich, T.; Fuchs, J. C.; Herrmann, A.; Kallenbach, A.; Maggi, C. F.; Neu, R.; Pütterich, T. et al. Compatibility of ITER scenarios with full tungsten wall in ASDEX Upgrade. Nucl. Fusion 2009, 49, 115014.

(7) Schweinzer, J.; Sips, A.; Tardini, G.; Schneider, P.; Fischer, R.; Fuchs, J.; Gruber, O.; Hobirk, J.; Kallenbach, A.; McDermott, R. et al. Confinement of 'improved H-modes' in the all-tungsten ASDEX Upgrade with nitrogen seeding. Nucl. Fusion 2011, 51, 113003. 
(8) Neuwirth, D.; Rohde, V.; Schwarz-Selinger, T.; ASDEX Upgrade Team, t. Formation of ammonia during nitrogen-seeded discharges at ASDEX Upgrade. Plasma Phys. Controlled Fusion 2012, 54, 085008.

(9) Oberkofler, M.; Douai, D.; Brezinsek, S.; Coenen, J. W.; Dittmar, T.; Drenik, A.; Romanelli, S. G.; Joffrin, E.; McCormick, K.; Brix, M. et al. First nitrogen-seeding experiments in JET with the ITER-like Wall. J. Nucl. Mater. 2013, 438, S258-S261.

(10) Rohde, V.; Oberkofler, M. Ammonia production in nitrogen seeded plasma discharges in ASDEX Upgrade. J. Nucl. Mater. 2015, 463, 672-675.

(11) Grunze, M.; Brundle, C. R.; Tomanek, D. Adsorption and decomposition of ammonia on a W(110) surface: Photoemission fingerprinting and interpretation of the core level binding energies using the equivalent core approximation. Surf. Sci. 1982, 119, 133-49.

(12) Egawa, C.; Naito, S.; Tamaru, K. Adsorption and decomposition of ammonia on W(100); XPS and UPS studies. Surf. Sci. 1983, 131, 49-60.

(13) Reed, A. P. C.; Lambert, R. M. Mechanism of ammonia decomposition on (100) oriented polycrystalline tungsten and single-crystal tungsten(100). J. Phys. Chem. 1984, 88, 19541959.

(14) Alnot, P.; Cassuto, A.; King, D. A. Decomposition (and synthesis) of ammonia on W\{100\}. A thermal molecular beam study. Faraday Discuss. Chem. Soc. 1989, 87, 291-302.

(15) Masson, D. P.; Merrill, R. P.; Houston, P. L. The sticking and dissociation of NH3 on W(110): a three-state model. Surf. Sci. 1995, 330, 239-254.

(16) Estrup, P. J.; Anderson, J. Adsorption and Decomposition of Ammonia on a Single-Crystal Tungsten (100) Surface. J. Chem. Phys. 1968, 49, 523-528.

(17) May, J. W.; Szostak, R. J.; Germer, L. H. Thermal breakup of NH3 adsorbed on W(211). Surf. Sci. 1969, 15, 37-76. 
(18) de Castro, A.; Alegre, D.; Tabares, F. L. Physisorption of ammonia on AISI 304L stainless steel at different surface temperature under high vacuum conditions. Nucl. Mater. Energy 2016, 9, 1-5.

(19) Yaala, M. B.; Marot, L.; Steiner, R.; Moser, L.; Temmerman, G. D.; Porosnicu, C.; Lungu, C. P.; Oberkofler, M.; Meyer, E. Quartz micro-balance and in situ XPS study of the adsorption and decomposition of ammonia on gold, tungsten, boron, beryllium and stainless steel surfaces. Nucl. Fusion 2018, 58, 106012.

(20) Luntz, A. C. In The Dynamics of Making and Breaking Bonds at Surfaces Chemical Bonding at Surfaces and Interfaces; A. Nilsson, L. G. M. P., Nørskov, J. K., Eds.; Elsevier: Amsterdam, 2008; Chapter 3, pp 143-254.

(21) King, D. A.; Wells, M. G. Molecular beam investigation of adsorption kinetics on bulk metal targets: Nitrogen on tungsten. Surf. Sci. 1972, 29, 454-482.

(22) Berenbak, B.; Butler, D. A.; Riedmuller, B.; Papageorgopoulos, D. C.; Stolte, S.; Kleyn, A. W. Sticking probability measurement in a reactive system. Surf. Sci. 1998, 414, $271-278$.

(23) Hodille, E.; Ghiorghiu, F.; Addab, Y.; Zaloznik, A.; Minissale, M.; Piazza, Z.; Martin, C.; Angot, T.; Gallais, L.; Barthe, M.-F. et al. Retention and release of hydrogen isotopes in tungsten plasma-facing components: the role of grain boundaries and the native oxide layer from a joint experiment-simulation integrated approach. Nucl. Fusion 2017, 57, 076019.

(24) Kisliuk, P. The Sticking Probabilities of Gases Chemisorbed on the Surfaces of Solids. J. Phys. Chem. Solids 1957, 3, 95-101.

(25) Cassuto, A.; King, D. A. Rate expressions for adsorption and desorption kinetics with precursor states and lateral interactions. Surf. Sci. 1981, 102, 388-404. 
(26) Arumainayagam, C. R.; McMaster, M. C.; Madix, R. J. Coverage dependence of molecular adsorption dynamics: ethane on platinum (111). J. Phys. Chem. 1991, 95, 2461-2465.

(27) Grunze, M.; Dowben, P. A.; Brundle, C. R. Ammonia cluster formation and NH3 decomposition on a Ni(100) surface. Surf. Sci. 1983, 128, 311-324.

(28) Rettner, C. T.; Mullins, C. B. Dynamics of the chemisorption of O2 on Pt(111): Dissociation via direct population of a molecularly chemisorbed precursor at high incidence kinetic energy. J. Chem. Phys. 1991, 94, 1626-1635.

(29) Walker, A. V.; Klötzer, B.; King, D. A. Dynamics and kinetics of oxygen dissociative adsorption on Pt110(1x2). J. Chem. Phys. 1998, 109, 6879-6888.

(30) Gee, A. T.; Hayden, B. E. The dynamics of O2 adsorption on Pt(533): Step mediated molecular chemisorption and dissociation. J. Chem. Phys. 2000, 113, 10333-10343.

(31) Jacobse, L.; den Dunnen, A.; Juurlink, L. B. F. The molecular dynamics of adsorption and dissociation of $\mathrm{O} 2$ on $\mathrm{Pt}(553)$. J. Chem. Phys. 2015, 143, 014703. 


\section{TOC Graphic}

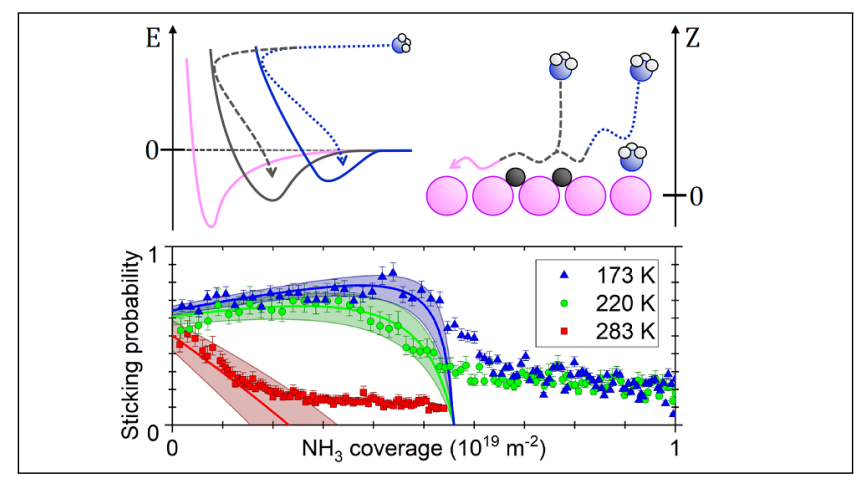

